\title{
Transcriptomic response is more sensitive to water deficit in shoots than roots of Vitis riparia (Michx.)
}

Vedbar Singh Khadka ${ }^{1,2}$, Kimberley Vaughn ${ }^{1}$, Juan Xie ${ }^{1,3}$, Padmapriya Swaminathan ${ }^{1,3}$, Qin Ma ${ }^{1,3}$, Grant R. Cramer ${ }^{4}$ and Anne Y. Fennell ${ }^{1,3^{*}}$ (i)

\begin{abstract}
Background: Drought is an important constraint on grapevine sustainability. Vitis riparia, widely used in rootstock and scion breeding, has been studied in isolated leaf drying response studies; however, it is essential to identify key root and shoot water deficit signaling traits in intact plants. This information will aid improved scion and rootstock selection and management practices in grapevine. RNAseq data were generated from $V$. riparia roots and shoots under water deficit and well-watered conditions to determine root signaling and shoot responses to water deficit.

Results: Shoot elongation, photosynthetic rate, and stomatal conductance were significantly reduced in water deficit (WD) treated than in well-watered grapevines. RNAseq analysis indicated greater transcriptional differences in shoots than in roots under WD, with 6925 and 1395 genes differentially expressed, respectively (q-value < 0.05). There were 50 and 25 VitisNet pathways significantly enriched in WD relative to well-watered treatments in grapevine shoots and roots, respectively. The ABA biosynthesis genes beta-carotene hydroxylase, zeaxanthin epoxidase, and 9-cis-epoxycarotenoid dioxygenases were up-regulated in WD root and WD shoot. A positive enrichment of ABA biosynthesis genes and signaling pathways in WD grapevine roots indicated enhanced root signaling to the shoot. An increased frequency of differentially expressed reactive oxygen species scavenging (ROS) genes were found in the WD shoot. Analyses of hormone signaling genes indicated a strong ABA, auxin, and ethylene network and an ABA, cytokinin, and circadian rhythm network in both WD shoot and WD root.

Conclusions: This work supports previous findings in detached leaf studies suggesting ABA-responsive binding factor 2 (ABF2) is a central regulator in ABA signaling in the WD shoot. Likewise, ABF2 may have a key role in $V$. riparia WD shoot and WD root. A role for $A B F 3$ was indicated only in WD root. WD shoot and WD root hormone expression analysis identified strong ABA, auxin, ethylene, cytokinin, and circadian rhythm signaling networks. These results present the first $A B A$, cytokinin, and circadian rhythm signaling network in roots under water deficit. These networks point to organ specific regulators that should be explored to further define the communication network from soil to shoot.
\end{abstract}

Keywords: Drought, Grapevine, ABA, ABF2, ABF3, NAC, Circadian rhythm, Cytokinin, Signaling network, Vitis riparia

\footnotetext{
* Correspondence: anne.fennell@sdstate.edu

${ }^{1}$ McFadden BioStress Laboratory, Agronomy, Horticulture, and Plant Science

Department, South Dakota State University, Brookings, SD 57006, USA

${ }^{3}$ South Dakota State University, Brookings, SD 57006, USA

Full list of author information is available at the end of the article
}

(c) The Author(s). 2019 Open Access This article is distributed under the terms of the Creative Commons Attribution 4.0 International License (http://creativecommons.org/licenses/by/4.0/), which permits unrestricted use, distribution, and reproduction in any medium, provided you give appropriate credit to the original author(s) and the source, provide a link to the Creative Commons license, and indicate if changes were made. The Creative Commons Public Domain Dedication waiver (http://creativecommons.org/publicdomain/zero/1.0/) applies to the data made available in this article, unless otherwise stated. 


\section{Background}

Sustainability of woody temperate fruit crops production has become a major concern in today's context of continued reduction of arable land and water resources due in part to an increase in global warming. The perennial grapevine is the most widely cultivated woody fruit crop with over 7.6 million hectares and 75 million metric tons production [1]. Vitis riparia Michx., the only grape species native to the upper Midwest region of the United States [2], plays a vital role in the global grape industry through its use in rootstocks, which are used commercially throughout the world as well as in hybrid scion cultivar development. $V$. riparia is noted for its resistance to phylloxera, adaptation to variant soil types, and low temperature tolerance, but is suggested to have limited drought tolerance relative to other grapevine genotypes [3-7]. With the changing climatic conditions, drought is a primary environmental constraint on grapevine growth, development, and sustainability [8-11].

Physiological responses to water deficit have been well studied in grapevine, and recently transcriptional changes have been examined in leaf tissues under a variety of water deficit conditions [12-16]. Rootstock and scion experiments have shown that increased root vigor and hydraulic conductance results in increased leaf area and leaf-area specific transpiration [16]. Root perception of soil water status promotes changes in gene expression and hydraulic conductance promoting ABA biosynthesis and signaling in plants [17-19]. Transcriptomic analysis of leaves and roots of drought tolerant and intolerant rootstocks indicate differences in leaf response are genotype dependent [18]. Signals from the root regulate stomata closure, which restricts water loss and limits carbon assimilation [20-25]. The synthesis of ABA and ethylene in the drying root are indicated as long-distance chemical signals to the shoot $[15,17,19,24]$. While many studies have addressed grapevine leaf and berry molecular responses under water deficit pointing to the central role of ABA in signaling response [13, 14, 26-33], there has been more limited exploration of root signaling characteristics $[17,18]$. However, these studies together indicate key roles for $\mathrm{ABA}$ and ethylene in grapevine responses to water deficit. We hypothesize that while ABA signaling plays a key role in the complex signaling between the root and shoot, the signaling networks will also include differences due to organ functional differences. In addition, it is expected that root response to water deficit is dependent on carbohydrate metabolism for osmotic potential changes where the carbohydrates are provided in large part by shoot photosynthetic activity. It is therefore essential to determine both the root signaling of soil water conditions and shoot responses to provide a well-informed genomic knowledge base for improved selection of grapevine rootstock and scion cultivars. The roots are the source of water uptake and signals of soil water conditions; therefore, both root and shoot need to be studied together. In this study, $V$. riparia root and shoot responses to a gradually induced water deficit were evaluated to identify signature tissue gene expression responses. The foundational information on root signaling of water deficit for this important rootstock species will be useful as we hypothesize that there will be differences in the root and green shoot signaling responses. This information will help identify key signaling networks and traits for improving grapevine management practices and selection of new rootstocks [30].

\section{Methods}

\section{Grapevine material and growth conditions}

Potted spur-pruned, ten-year-old V. riparia Michx. (U.S. National Plant Germplasm System PI588259) vines were removed from cold storage, root pruned, repotted in fresh medium in $15 \mathrm{~L}$ pots, and grown under a long photoperiod (15 h) with $25 / 20^{\circ} \mathrm{C} \pm 3{ }^{\circ} \mathrm{C}$ day/night temperatures and 600 to $1400 \mu \mathrm{mol} \mathrm{m} \mathrm{m}^{-2} \mathrm{~s}^{-1}$ photosynthetic photon flux in a climate-controlled, un-shaded glass greenhouse (En Tech Control Systems Inc., Montrose, MN) in Brookings, SD, USA $\left(44.3^{\circ} \mathrm{N}\right)$. Three shoots were allowed to develop on each grapevine. After $30 \mathrm{~d}$, when the grapevine shoots had reached 12-15 nodes, 36 grapevines were randomized into two groups: water deficit (WD) and well-watered control (C). Five days after randomization, differential water treatments began: WD received no water and $C$ received $2 \mathrm{~L}$ of tap water each day, which allowed for gravitational water runoff. Shoot elongation (length and node number) was recorded in nine grapevines from each treatment every other day, starting the day before treatment induction. Physiological measurements occurred at 7 and $14 \mathrm{~d}$ of treatment on three separate replicates for each treatment; each separate replicate was composed of three vines.

\section{Physiological measurements}

Stem water potential ( $\left.\Psi_{\text {stem }}\right)$ was measured with a modified 3005HGPL plant water status console (Soil Moisture Equipment Corp., Santa Barbara, CA) on young, newly expanded leaves (5th fully expanded leaf from the shoot tip) as previously described [31]. The leaves were wrapped in foil and sealed in a plastic bag while still on the grapevine to equilibrate to the stem water potential [34]. The leaves equilibrated for two hours, were excised, and $\Psi_{\text {stem }}$ was immediately measured with the pressure chamber. One leaf from each grapevine in each replicate was measured; vines were only used once for these destructive measurements, thus no repeat measures were made.

Net photosynthesis rate $(\mathrm{Pn})$ and stomatal conductance (SC) were measured with a CI-340 portable photosynthesis meter (CID, Inc.) just prior to measuring stem 
water potential $\left(\Psi_{\text {stem }}\right)$. Gas exchange measurements were taken using leaves on separate shoots, but at the same node position as the leaves used for $\Psi_{\text {stem. }}$. Photosynthetic measurements were made in one grapevine from each replication for each treatment (WD, C) and time point $(7 \mathrm{~d}, 14 \mathrm{~d})$. The leaf chamber covered $11 \mathrm{~cm}^{2}$ of the total leaf area and the meter operated under open conditions. Leaf and air temperature were measured by an additional sensor. A CI-301LA light attachment was used to maintain photosynthetically active radiation (PAR) at $\geq 2400 \mu \mathrm{mol} \mathrm{m}^{-2} \mathrm{~s}^{-1}$. Fresh weight (FW) measurements were recorded from each grapevine on a 4-node shoot tip and $5 \mathrm{~cm}$ root tips for each of the three separate replicates $(n=3)$. The organs were then dried at $60^{\circ} \mathrm{C}$ and dry weight (DW) recorded. Percent water content (WC), hydration value ((HV: g H2O/g DW; $(W C=(F W-D W) /$ $\mathrm{DW})$ ) and dry matter content (DMC: DW/FW) were calculated. At the same time, root tips $(2 \mathrm{~cm})$ and 4-node shoot tips were excised, plunged in liquid nitrogen, and stored at $-80^{\circ} \mathrm{C}$ for future RNA extraction.

Statistical significance of treatment, time, and interaction effects of Pn, SC, $\Psi_{\text {stem }}$, and WC values were determined using analysis of variance (ANOVA). These measures were conducted using new vines at each time point as water potential and water content were destructive measures. Mean separation of significant factors was determined with a Student-Newman-Keuls test at $p<0.05$. Growth measurements were analyzed with repeated measure ANOVA as the same vines were measured repeatedly throughout the study.

\section{RNA extraction}

Root and shoot samples from the $14 \mathrm{~d} \mathrm{C}$ and WD treatments were used for transcriptomic analysis, as this time point showed distinct physiological differences between treatments. Total RNA was extracted from $100 \mathrm{mg}$ of frozen pulverized shoot tips as described by [35]. Total RNA was extracted from multiple root tips $(5 \mathrm{~cm})$ for each replicate using the Qiagen RNeasy Midi RNA isolation kit (Qiagen, 75,144) according to manufacturer protocol. DNA was removed using an RNase-Free DNase kit (Qiagen, \#79254). RNA quality and quantity of the shoot and root tissue were verified using an Agilent 2100 Bioanalyzer and RNA 6000 nano chip (\#5067-1511).

\section{Real-time PCR}

Real-time PCR was used to evaluate expression levels of seven ABA metabolic and signaling genes and a reference gene in root and shoot tissues prior to RNA sequencing. Primers were designed with PrimerQuest (Integrated DNA Technologies, http://www.idtdna.com), using the default parameters for real-time PCR (Additional file 1: Table S1). Candidate genes included zeaxanthin epoxidase
(ABA1; VIT_07s0031g00620), 9-cis-epoxycarotenoid dioxygenase 3 (NCED3; VIT_19s0093g00550), protein phosphatase 2CA (PP2CA; VIT_13s0019g02200), ABA 8'-hydroxylase 3 (CYP707A3; VIT_02s0087g00710), molybdenum cofactor sulfurase (ABA3, SIR3; VIT_19s0027g01090), protein phosphatase 2C 16 (ABI1; VIT_11s0016g03180), and UDP-glycosoyltransferase 73B3 (UGT73B3; VIT 03s0063g00040). V. riparia eukaryotic initiation factor4A (VreIF4A) was used as a reference gene [35] (transcript abundance of this gene was not affected by the water deficit treatment in our RNAseq dataset). Three separate PCR reactions were conducted for each of the three replicates from each treatment. Primer optimization, cDNA synthesis, standard curves, and real-time PCR reactions (including parameters) were conducted as described by [35]. Data analysis was performed with MxProQPCR software (Stratagene, LaJolla, CA, USA). Relative expression ratios of candidate genes (ABA1, NCED3, PP2CA, CYP707A3, ABA3, ABI1, and UGT737B3) to the reference gene (VreIF4A) were calculated using $\mathrm{R}=\left(\mathrm{E}_{\text {target }}\right)^{\Delta \mathrm{Ct}}$ target (control-sample) $/\left(\mathrm{E}_{\mathrm{ref}}\right)^{\Delta \mathrm{Ct}}$ ref (control-sample); where $\mathrm{E}=1+$ MxPro efficiency; target $=$ candidate gene; ref. $=$ VreIF4A; control = C (well-watered grapevines), [36] For direct comparison between tissues, all genes were expressed relative to their specific well-watered control. A Student's t-test, $p$-value $\leq 0.05$ was used to determine significant gene expression differences between root and shoot tissue for individual genes.

\section{RNA sequencing and analysis}

RNAseq was performed at Cornell University Life Sciences Core Laboratories (Ithaca, NY, USA). Bar coded libraries were prepared for three separate replicates of each treatment and organ (root or shoot; two tissues, two water treatments with three replicates for each $=12$ separate libraries) and they were sequenced using an Illumina $^{\circ}$ HiSeq $^{\text {tm }} 2000$ Sequencing System (Illumina, Inc., San Diego, CA, USA). Illumina sequences from each of WD and C treatments for each organ (root and shoot) were generated as $100 \mathrm{bp}$ single-end reads in FASTQ format. Quality of sequences were explored with FASTX toolkit and sequences cleaned using Prinseq [37]. The cleaning procedure included, trimming low quality reads from the ends to a Phred quality score $>20$ and filtering reads with a length less than $20 \mathrm{bp}$. Samples after cleaning had high quality reads (20 to100 bp).

Bowtie2 V2.1.0 was used to build the index of the grapevine reference genome assembly (PN40024 12X, V1), a nearly homozygous inbred of the $V$. vinifera Pinot Noir cultivar. TopHat V2.0.8 was used to map each of the cleaned samples to the Bowtie build index and Cufflinks V2.1.1 was used to quantify transcript abundance in terms of Reads Per Kilobase of exon model per Million mapped reads (RPKM). SAMtools V0.1.18 was used for sequence manipulation and the htseq-count 
script in the HTSeq package was used to count reads mapped to the grapevine gene models. Differential gene expression of the WD treatment relative to the $\mathrm{C}$ was determined using the Cuffdiff program within Cufflinks with default parameters. In significantly differentially expressed genes (DEGs) at a false discovery rate (FDR)-adjusted $P$-value (q-value) $<0.05$, the terms up- or down-regulated will be used to refer to the expression values of the WD root relative to $C$ root expression values or WD shoot expression values relative to $C$ shoot expression values. The datasets generated for this study can be found in the NCBI sequence read archive under accession \#GSE109065, SRA SRP130959.

\section{VitisNet pathway and gene set enrichment analysis}

Gene Set Enrichment Analysis (GSEA) was conducted using read count data from each of the three replicates for each treatment using GSEA-P 2.0 (http://www.broad. mit.edu/GSEA) and 247 VitisNet pathways (https:// openprairie.sdstate.edu/vitisnet-12x_files/) including at least 7 genes [38-42]. The recommended GSEA-P 2.0 default parameters of 1000 permutations, nominal $p$-value $<0.05$ and FDR q-value $<0.25$ were used to identify enriched molecular pathways related to WD or C [39]. Positive significantly enriched pathways are generally up-regulated in WD root or shoot relative to their specific C organ and negatively enriched pathways are down-regulated in WD relative to the respective $\mathrm{C}$ organ.

\section{Network construction for hormone DEGs in root and shoot}

The expression values of DEGs that belong to target functions (i.e., ABA, auxin, circadian rhythm, cytokinin, and ethylene) were extracted from the replicated root or shoot gene expression matrix. Then the Pearson correlation coefficients (PCCs) between genes were calculated in R. The PCCs between a gene and itself were assigned zero, as self-correlation was not considered in this study. Next, the correlation matrix was modeled as a network using the graph_from_adjacency_matrix function in the igraph R package (https://www.r-project.org/), in which nodes representing genes and weight of edges connecting two genes being the PCCs between the two genes. Only the edges with an absolute weight greater than cutoff for a network were kept in our analysis (ABA, cytokinin, and circadian rhythm networks $(0.8$ for root and 0.96 for shoot) and ABA, auxin, and ethylene networks (0.96 for root and shoot)). The four generated networks (two each for roots and shoots) were exported as the GML-format files. These files were visualized using Cytoscape (version 3.4.0; www.cytoscape.org) with node size representing degree, edge width reflecting correlation, and node colors to distinguish hormone subnetworks. The topology of these networks follows the Group Attributes Layout option in Cytoscape.

\section{Results \\ Water deficit reduced shoot elongation and photosynthesis}

WD grapevines had a lower stem water potential $\left(\Psi_{\text {stem }}\right)$ than the controls (Table 1) and there was a strong correlation $(r=0.90)$ between $\Psi_{\text {stem }}$ and photosynthesis $(\mathrm{Pn})$. The $\mathrm{Pn}$ and stomatal conductance (SC) were significantly lower in WD grapevines relative to the control grapevines.

Shoot elongation and node number were significantly reduced in the WD grapevines in comparison to the control grapevines after $10 \mathrm{~d}$ (Fig. 1). Water deficit effects were significant for percent water content (\%WC) and dry matter content in shoot tip and root; however, there was not a significant interaction between time and treatment (Additional file 2: Table S2). A small amount of leaf shedding from the base of the WD shoot was observed after $14 \mathrm{~d}$ of water deficit. Visual and physical root changes were observed at $14 \mathrm{~d}$ of water deficit. Roots were brown, stiffer (not easily bent), and there were fewer roots visible on the exterior of the root ball for the WD grapevines compared to $\mathrm{C}$ grapevines (Fig. 2a-b). Shoot tips were similar between both treatments (Fig. 2c-d).

\section{Distinct root and shoot transcriptome profiles were revealed under water deficit treatment}

DEGs in WD roots or WD shoots were further identified as up- or down-regulation relative to their respective $C$ treatment. There were fewer DEGs in WD root (1385) than in the WD shoot (6925) tissues (Additional file 3: Table S3, Additional file 4: Table S4). In the WD root there were 885 up-regulated and 500 down-regulated DEGs, whereas in the WD shoot there was a more similar number of up- and down-regulated DEGs (2978 and 3947, respectively) (Additional file 3: Table S3, Additional file 4: Table S4). Between the root and shoot there were 836 DEGs in common ((Additional file 3: Table S3,

Table 1 Leaf physiological changes in response to water deficit

\begin{tabular}{|c|c|c|c|c|}
\hline \multirow{2}{*}{$\begin{array}{l}\text { Treatment water } \\
\text { status }\end{array}$} & \multirow{2}{*}{$\begin{array}{l}\text { Treatment } \\
\text { day }\end{array}$} & \multirow{2}{*}{$\begin{array}{l}\operatorname{Pn}(\mathrm{z}) \\
\mathrm{mmol} \mathrm{m}{ }^{-2} \mathrm{~s}^{-1}\end{array}$} & \multirow{2}{*}{$\begin{array}{l}\mathrm{SC} \\
\mathrm{mmol} \mathrm{m}^{-2} \mathrm{~s}^{-1}\end{array}$} & \multirow{2}{*}{$\begin{array}{l}\Psi_{\text {stem }}(\mathrm{z}) \\
\mathrm{MPa}\end{array}$} \\
\hline & & & & \\
\hline C & 7 & 6.76 & @ & -0.39 \\
\hline C & 14 & 4.99 & 14.98 & -0.34 \\
\hline WD & 7 & 1.72 & @ & -1.02 \\
\hline WD & 14 & 1.83 & 0.57 & -1.27 \\
\hline
\end{tabular}

Mean \pm SE of net photosynthesis rate (Pn), stomatal conductance (SC) and stem water potential $\left(\Psi_{\text {stem }}\right)$. Two-way ANOVA significant main effects for treatment is noted with $z,(p \leq 0.05, n=3)$; there were no significant time or time $x$ treatment interactions, @ indicates not measured 


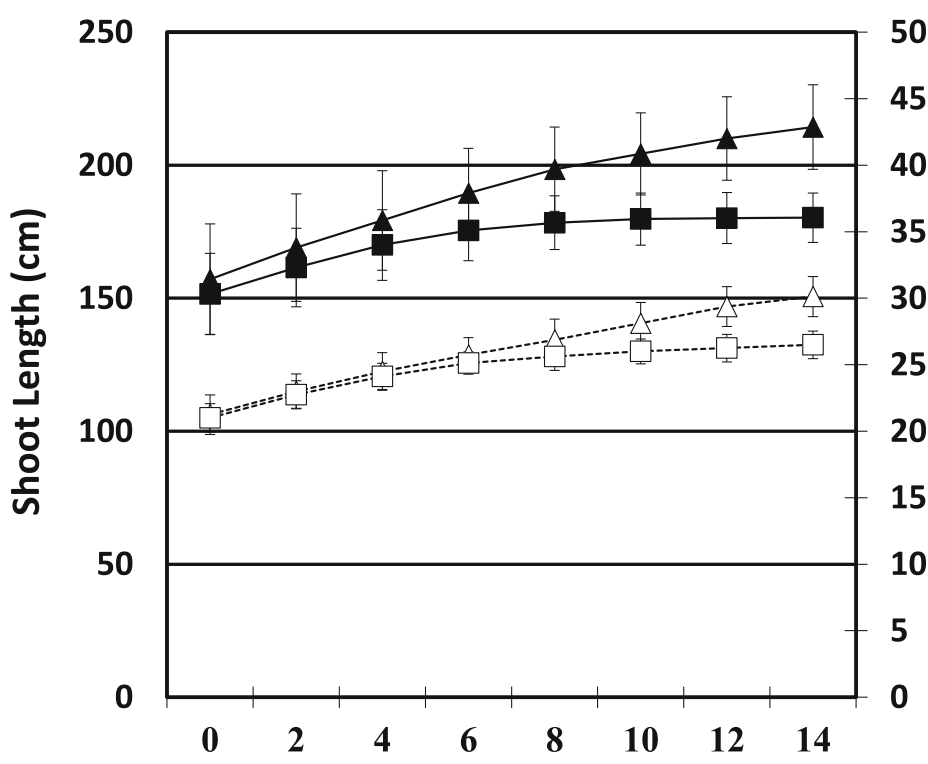

Fig. 1 Primary shoot length and node number. Water deficit (WD, squares) and control (C, triangle). Solid lines indicate primary shoot length; dashed lines represent node number

Additional file 4: Table S4); DEGs in both organs are in bold in the tables). The majority of the DEGs in common had a similar up-regulated pattern in WD root (560) and WD shoot (522), with only 73 that had opposite regulation in WD root and WD shoot (Additional file 5: Figure S1).

Seven candidate genes (ABA1, NCED3, PP2CA, $C Y P 707 A 3, A B A 3, A B I 1$, and UGT737B3) in the ABA biosynthesis and catabolism pathways examined by real time $\mathrm{PCR}$ were expressed as a fold-change of $\mathrm{WD} / \mathrm{C}$ of the

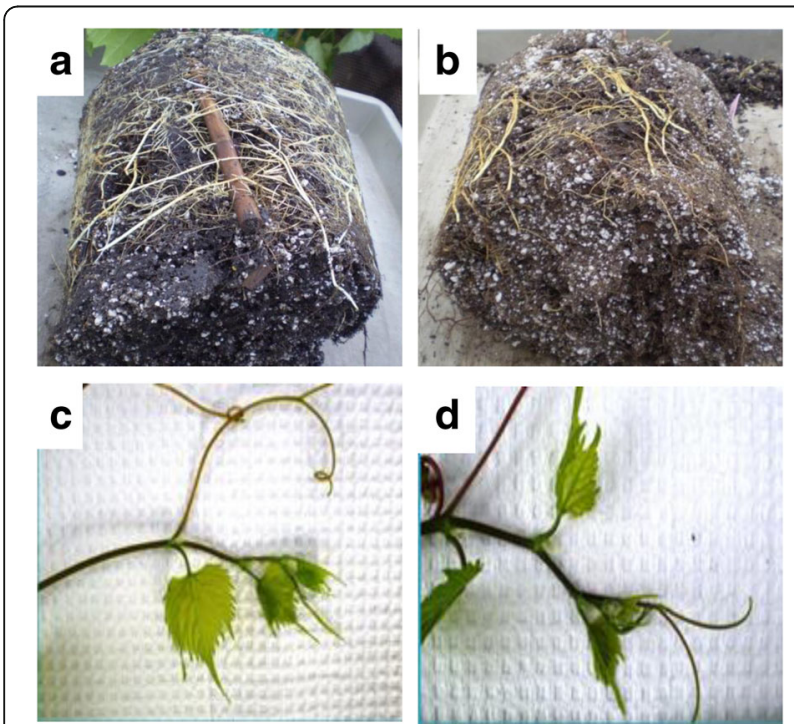

Fig. 2 V. riparia root ball and shoot tip. a Well-watered control root; b Water deficit root; c Well-watered control shoot tip; $\mathbf{d}$ Water deficit shoot tip respective organ (Additional file 6: Figure S2). The UDP-glycosyltransferase (UGT737B3, VIT_03s0063g00040) selected was similar to an Arabidopsis UDP-glycosyltransferase ortholog, that is specifically expressed in Arabidopsis roots (At4g34135); however, it was not expressed in $V$. riparia roots in PCR reactions and not significant in RNAseq analysis. NCED3 showed significantly greater transcript abundance in WD roots than in WD shoots ( $p$-value $=0.003$ ) (Additional file 6: Figure S2). In contrast, CYP707A3 had significantly greater fold-change in WD shoot (p-value $=0.019$ ). There was no difference in the WD/C for $A B A 1, P P 2 C A, A B A 3$, and $A B I 1$ by PCR analysis between root and shoot. A strong correlation (an average of $\mathrm{r}=0.99$ ) was observed for the expression fold-change of WD to $\mathrm{C}$ with real-time PCR and RNAseq expression data. The greater sensitivity of RNAseq analysis was apparent, with the detection of the significant up-regulation of PP2CA (VIT_13s0019g02200) and ABI1 (VIT_11s0016g03180) in WD root and WD shoot (Additional file 3: Table S3, Additional file 4: Table S4) which was not detected by PCR.

\section{A greater number of molecular pathways were} significantly enriched in WD shoot than in WD root

Gene set enrichment analysis using 247 VitisNet pathways indicated there were 25 and 50 VitisNet pathways significantly enriched (FDR q-value $<0.25$ ) in WD root and WD shoot, respectively (Table 2 ). There were eight VitisNet pathways positively enriched in both WD root and WD shoot (galactose and cyano-amino acid metabolism, photosynthesis, ABA biosynthesis, cytokinin 
Table 2 VitisNet pathway enrichment in water deficit root and shoot

\begin{tabular}{|c|c|c|c|c|c|c|}
\hline \multirow[t]{2}{*}{ Category } & \multirow[t]{2}{*}{ VitisNet pathway } & \multirow{2}{*}{$\begin{array}{l}\text { Pathway } \\
\text { size }\end{array}$} & \multicolumn{2}{|l|}{ Root } & \multicolumn{2}{|l|}{ Shoot } \\
\hline & & & NES & FDR q-val & NES & FDR q-val \\
\hline \multirow[t]{3}{*}{ 1.1 Carbohydrate Metabolism } & VV10052 Galactose Metabolism & 149 & 1.59 & 0.17 & 1.54 & 0.18 \\
\hline & VV10520 Nucleotide Sugars Metabolism & 61 & -1.48 & 0.30 & -1.88 & 0.01 \\
\hline & W10530 Aminosugars Metabolism & 79 & 1.10 & 0.90 & -1.64 & 0.09 \\
\hline \multirow[t]{3}{*}{ 1.2 Energy Metabolism } & VV10195 Photosynthesis & 114 & 1.74 & 0.09 & 1.13 & 0.47 \\
\hline & W10196 Photosynthesis Antenna Proteins & 19 & 1.99 & 0.00 & -0.86 & 1.00 \\
\hline & VV10680 Methane Metabolism & 113 & 1.56 & 0.17 & -1.60 & 0.11 \\
\hline \multirow[t]{2}{*}{ 1.3 Lipid Metabolism } & VV10061 Fatty Acid Biosynthesis & 70 & -1.83 & 0.09 & -1.64 & 0.09 \\
\hline & W10561 Glycerolipid Metabolism & 144 & 1.09 & 0.91 & 1.55 & 0.20 \\
\hline \multirow[t]{3}{*}{ 1.5 Amino Acid Metabolism } & W10360 Tyrosine Metabolism & 147 & 1.12 & 0.88 & -1.46 & 0.23 \\
\hline & W10400 Phenylalanine Metabolism & 200 & 0.95 & 0.97 & -1.71 & 0.06 \\
\hline & $\begin{array}{l}\text { W10400 Phenylalanine Tyrosine \& Tryptophan } \\
\text { Biosynthesis }\end{array}$ & 143 & 1.12 & 0.88 & -1.55 & 0.16 \\
\hline \multirow[t]{2}{*}{ 1.6 Other Amino Acid Metabolism } & VV10460 Cyanoamino Acid Metabolism & 34 & 1.60 & 0.16 & 2.00 & 0.02 \\
\hline & W10480 Glutathione Metabolism & 131 & 1.26 & 0.58 & 2.23 & 0.00 \\
\hline 1.7 Glycan Biosynthesis \& Metabolism & W10511N-Glycan Degradation & 65 & 0.85 & 1.00 & -1.67 & 0.08 \\
\hline \multirow[t]{2}{*}{ 1.8 Cofactors \& Vitamin Metabolism } & W10790 Folate Biosynthesis & 30 & 1.24 & 0.62 & 1.56 & 0.20 \\
\hline & W10860 Porphyrin and Chlorophyll Metabolism & 64 & 1.60 & 0.17 & 1.18 & 0.52 \\
\hline \multirow{7}{*}{$\begin{array}{l}\text { 1.9 Biosynthesis of Secondary } \\
\text { Metabolites }\end{array}$} & W10900 Terpenoid Biosynthesis & 156 & 0.93 & 0.99 & -1.53 & 0.18 \\
\hline & W10902 Monoterpenoid Biosynthesis & 181 & 1.46 & 0.29 & 1.61 & 0.18 \\
\hline & W10904 Diterpenoid Biosynthesis & 68 & -1.18 & 0.72 & -1.45 & 0.23 \\
\hline & W10906 Carotenoid Biosynthesis & 41 & 1.29 & 0.52 & 2.05 & 0.02 \\
\hline & W10940 Phenylpropanoid Biosynthesis & 222 & 0.68 & 1.00 & -1.80 & 0.03 \\
\hline & W11002 Auxin Biosynthesis & 90 & 1.20 & 0.69 & 1.58 & 0.20 \\
\hline & VV11013 ABA Biosynthesis & 16 & 1.88 & 0.02 & 1.98 & 0.02 \\
\hline 1.10 Other Metabolism & VV11000 Single Reactions & 167 & 1.54 & 0.18 & -1.57 & 0.14 \\
\hline 2.4 Replication and Repair & W23030 DNA Replication & 64 & -1.40 & 0.36 & -1.63 & 0.09 \\
\hline \multirow[t]{4}{*}{ 3.2 Hormone Signaling } & W30003 ABA Signaling & 151 & 1.61 & 0.17 & 1.15 & 0.53 \\
\hline & W30007 Auxin Signaling & 271 & 0.98 & 0.97 & -2.03 & 0.00 \\
\hline & VV30007 Cytokinin Signaling & 68 & 1.56 & 0.19 & 1.46 & 0.25 \\
\hline & VV30010 Gibberellin Signaling & 36 & -1.93 & 0.07 & -1.61 & 0.11 \\
\hline \multirow[t]{2}{*}{ 3.3 Plant-specific Signaling } & W34627 R Proteins from Plant Pathogen Interaction & 349 & 0.71 & 1.00 & -2.03 & 0.00 \\
\hline & Circadian Rhythm & 64 & 1.58 & 0.17 & 1.15 & 0.52 \\
\hline \multirow[t]{2}{*}{ 4.1 Transport and Catabolism } & W44140 Regulation of Autophagy & 26 & 1.56 & 0.16 & 1.55 & 0.18 \\
\hline & VV44145 Phagosome & 118 & -1.80 & 0.09 & -1.45 & 0.23 \\
\hline 4.2 Cell Motility & W44180 Regulation of Actin Cytoskeleton & 343 & -0.96 & 1.00 & -2.07 & 0.00 \\
\hline \multirow[t]{2}{*}{ 4.3 Cell Growth and Death } & W40006 Cell Wall & 454 & 1.05 & 0.94 & -1.72 & 0.07 \\
\hline & W44110 Cell Cycle & 323 & -0.96 & 1.00 & -2.16 & 0.00 \\
\hline 5.1 Membrane Transport & W52010 ABC Transporters & 239 & 0.98 & 0.98 & 1.49 & 0.22 \\
\hline 5.2 Hormone Transport & W50004 Auxin Transport & 52 & 1.13 & 0.86 & -1.69 & 0.07 \\
\hline 5.3 Transport System & W50113 Thylakoid Targeting Pathway & 48 & 1.72 & 0.07 & -1.22 & 0.49 \\
\hline \multirow[t]{3}{*}{ 5.4 Transporter Catalog } & W50105 Transport Electron Carriers & 61 & 1.74 & 0.07 & -0.65 & 1.00 \\
\hline & W50122 Porters Cat 7 to 17 & 237 & 1.32 & 0.51 & -1.44 & 0.23 \\
\hline & W50135 Primary Active Transporter Cat D2 to E2 & 81 & 1.16 & 0.80 & 1.84 & 0.04 \\
\hline
\end{tabular}


Table 2 VitisNet pathway enrichment in water deficit root and shoot (Continued)

\begin{tabular}{|c|c|c|c|c|c|c|}
\hline \multirow[t]{2}{*}{ Category } & \multirow[t]{2}{*}{ VitisNet pathway } & \multirow{2}{*}{$\begin{array}{l}\text { Pathway } \\
\text { size }\end{array}$} & \multicolumn{2}{|l|}{ Root } & \multicolumn{2}{|l|}{ Shoot } \\
\hline & & & NES & FDR q-val & NES & FDR q-val \\
\hline \multirow[t]{18}{*}{ 6.0 Transcription Factors } & W60003 AP2 EREBP & 137 & 1.34 & 0.50 & -1.49 & 0.20 \\
\hline & W60004 ARF & 29 & 0.79 & 1.00 & -1.48 & 0.21 \\
\hline & W60008 AUXIAA & 23 & 1.00 & 0.97 & -1.68 & 0.07 \\
\hline & W60011 BHLH & 148 & 1.24 & 0.61 & -1.72 & 0.07 \\
\hline & W60015 C2C2-DOF & 25 & 1.63 & 0.16 & -1.05 & 0.74 \\
\hline & W60019 C2C2-YABBY & 7 & 1.72 & 0.08 & -1.27 & 0.46 \\
\hline & W60022 CPP & 7 & 1.21 & 0.66 & -1.49 & 0.20 \\
\hline & W60029 G2-LIKE & 37 & 1.54 & 0.18 & 1.30 & 0.37 \\
\hline & W60033 GRF & 12 & -0.74 & 1.00 & -1.50 & 0.19 \\
\hline & W60044 MYB & 166 & 0.96 & 0.97 & -1.51 & 0.19 \\
\hline & VV60046 NAC & 75 & 1.65 & 0.14 & 1.88 & 0.04 \\
\hline & W60055 SBP & 20 & 1.26 & 0.57 & -1.51 & 0.19 \\
\hline & W60061 TCP & 19 & 0.89 & 1.00 & -1.52 & 0.19 \\
\hline & VV60070 ORPHANS CCT & 8 & -1.77 & 0.08 & -1.44 & 0.24 \\
\hline & W60073 ORPHANS ZF-B BOX & 15 & 1.27 & 0.55 & 1.87 & 0.041 \\
\hline & VV60077 OTHER ZF-AN1 & 13 & 1.53 & 0.19 & 1.74 & 0.08 \\
\hline & W60079 OTHER ZF-DHHC & 23 & -0.91 & 1.00 & -1.48 & 0.21 \\
\hline & W60082 GNAT & 36 & 1.56 & 0.18 & -0.89 & 0.99 \\
\hline
\end{tabular}

A positive normalized enrichment score (NES) indicates VitisNet pathways enriched in WD tissues and a negative NES indicates pathways enriched in C treatments at nominal $p$-value $<0.05$ and false discovery rate (FDR) $q$-value $<25 \%$. Bold indicates an enrichment in both root and shoot

signaling, autophagy, and transcription factors (no apical meristem/ataf1/2/cup-shaped cotyledon $(N A C)$ and other zinc finger-an1 (ZF-AN1)). Five pathways were negatively enriched in both WD root and WD shoot (nucleotide sugar metabolism, fatty acid biosynthesis, gibberellin signaling, phagosome, and transcription factor (ORPHAN CCT)).

Positively enriched pathways specific to WD root were ABA and circadian rhythm signaling, photosynthesis antenna proteins, porphyrin and chlorophyll metabolism, transport (thylakoid and electron carriers), and transcription factors (C2C2-dna-binding with one finger (C2C2-DOF), C2C2-YABBY, G2-Like, and gcn5-related n-acetyltransferases $(G N A T)$ ) (Table 2). A total of nine pathways (glycerol lipid and glutathione metabolism; folate, monoterpenoid, carotenoid, and auxin biosynthesis; ATP-binding cassette transporters (abc transporter) and primary active transporter; and orphan zinc finger-b box transcription factors) were positively enriched only in WD shoot. Conversely, canonic shoot pathways related to photosynthesis were enriched in root and not in the shoot. In addition, only two carbohydrate metabolism pathways were positively enriched in WD root and negatively enriched in WD shoot (methane and single reactions). A large number of pathways (26) were negatively enriched only in WD shoot, including pathways in carbohydrate and amino acid metabolism, secondary metabolite biosynthesis, DNA replication, cell wall and cycle, auxin transport, and multiple transcription factor families.

\section{ABA biosynthesis and catabolism pathways had similar expression profiles in WD root and WD shoot}

Two beta-carotene 3-hydroxylase 1 genes (VIT 02s0025g00240, VIT_16s0050g01090) upstream of ABA biosynthesis pathway were up-regulated in both WD root and WD shoot (Additional file 3: Table S3, Additional file 4: Table S4). In the first committed step of the ABA biosynthesis pathway, two differentially expressed NCED genes (VIT_10s0003g03750, VIT_19s0093g00550) were up-regulated in WD root and only the latter was differentially up-regulated in WD shoot. Abscisic acid aldehyde oxidase was not differentially expressed in either organ. UPD-glycosyltransferase (VIT_12s0055g00020), which promotes ABA conjugation to ABA-GE [43], was up-regulated in WD shoot. This can be reversed by beta glucosidase (VIT_01s0011g00760, VIT_17s0000g02680) which were up- and down-regulated respectively in WD shoot. Degradation of ABA to phaseic acid is initiated by ABA 8 '-hydroxylase (ABAHASE). Three putative ABAHASE genes (VIT_06s0004g05050, VIT_03s0063g00380, and VIT_02s0087g00710) were differentially up-regulated in WD shoot and/or WD root. 
A greater number of up-regulated $A B A$, cytokinin, and circadian rhythm signaling DEGs were found in WD shoot than in WD root

In WD roots, there were more auxin, $A B A$, cytokinin, ethylene, jasmonate, and circadian rhythm DEGs up-regulated than down-regulated. WD shoot had greater numbers of hormone related DEGs than WD root; however, more auxin and ethylene related DEGs were down-regulated rather than up-regulated.

In WD grapevine, a greater number of ABA signaling DEGs were found in WD shoot $(35,26)$ than in WD root $(20,4)$ (up-, down-regulated, respectively). The ABA signaling genes pyrabactin resistance1)/PYR1-like/ regulatory components of aba receptors (PYR/PYL/ $R C A R$ ) were down-regulated in WD root and shoot with the exception of PYL6 (VIT_16s0050g02620) in WD shoot (Fig. 3). In WD root, all protein phosphatase type 2C (PP2C) DEGs (5) were up-regulated, and in WD shoot, 13 of the 20 DEGs were up-regulated. A similar number of the serine/threonine kinases (SnRK2) DEGs were up-regulated in WD root (14) and WD shoot (15) and there were two SnRKs DEGs (VIT_15s0024g01670 and VIT_09s0002g03120) up-regulated in the WD root. The SnRKs phosphorylate a group of ABA-responsive element binding factors $(a b f \mathrm{~s}$, basic leucine zipper $(b Z I P)$ transcription factors) which activate downstream gene expression. In both the WD shoot and WD root, downstream genes $A B F 2$ and $A B F 3$ were up-regulated relative to its respective $C$ grapevine organ. Several other $b Z I P$ transcription factor genes were differentially expressed (Fig. 3); however, only two were up-regulated in both WD root and WD shoot (abscisic acid insensitive 1 and $5(A B I 1, A B I 5))$.

\section{Ethylene response transcription factors were up-regulated in root and shoot}

Ethylene signaling has been associated with water deficit in leaves [14] and in this study there were 83 DEGs out of the total 213 annotated as ethylene signaling genes [42], (34 up- and 49 down-regulated). Ethylene and auxin DEGs had a greater number of down-regulated genes than up-regulated genes in WD shoot. In WD root, 21 of 27 DEGs, predominately ethylene response transcription factors $(E R F)$, were up-regulated. Of these

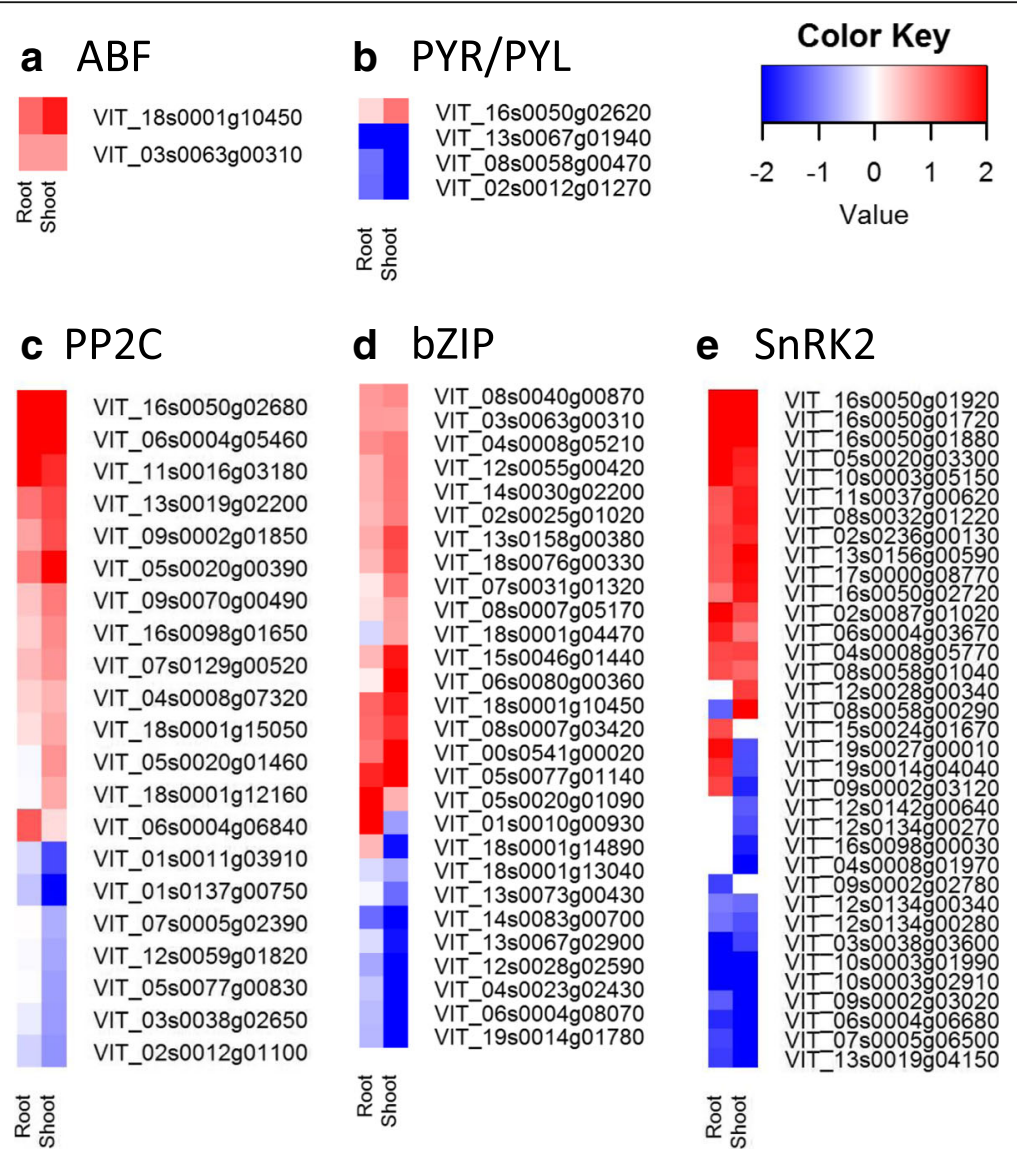

Fig. 3 ABA biosynthesis and catabolism related gene expression profiles. a ABF; b PYR/PYL; c PP2C; d bZIP and e SnRK2. Heat map values are fold change $\log _{2}$, the red and blue colors represent up- and down-regulation of the gene expression in water deficit tissue relative to their respect to control tissue in root and shoot 
only 14 were up-regulated in common between the WD root and WD shoot. Auxin and gibberellin signaling pathways were negatively enriched in WD root and WD shoot. In WD shoot, there were 165 DEGs out of the 467 total annotated auxin biosynthesis, signaling and transporter related genes [42], and 35 of these were up-regulated (predominately signaling genes, 21 total). In the WD root, there were only 29 auxin-related DEGs and 18 were up-regulated (15 signaling and 3 biosynthesis genes). Ten of these were up-regulated in common with those in WD shoot. In contrast to WD shoot, there were more auxin and ethylene DEGs up-regulated in WD root than were down-regulated.

\section{The cytokinin signaling pathway was positively enriched in water deficit shoots}

The cytokinin signaling pathway was positively enriched in both WD root and WD shoot. In WD shoot, there were 30 DEGs of the total 80 cytokinin signaling pathway genes with multiple type-A arabidopsis response regulators $(A R R s$, e.g. 5) and arabidopsis psuedo response regulators (APRR, e.g. 4) up-regulated in WD shoot (Additional file 3: Table S3, Additional file 4: Table S4). The majority of these were up-regulated (20) and involved in regulation of transcription. In contrast, only four genes were up-regulated in WD root and two of these were in common with the WD shoot. Of the 79 circadian rhythm pathway genes, there were a similar number of circadian rhythm DEGs up-regulated in WD root (10) and WD shoot (15) (Additional file 3: Table S3, Additional file 4: Table S4), with five of these DEGs in both WD shoot and WD root. WD root had only one down-regulated circadian rhythm DEGs (constans-like 14) and all other constans-like DEGs were up-regulated in both WD shoot and WD root.

\section{ABA, cytokinin, and circadian rhythm networks identified in water deficit root and shoot}

Networks were constructed for ABA, auxin, and ethylene or $A B A$, cytokinin, and circadian rhythm related DEGs. There was a greater number of DEGs with a high correlation between $\mathrm{ABA}$ and ethylene than between $\mathrm{ABA}$ and auxin in both root and shoot (Fig. 4a, b). ABF2 is strongly correlated with ethylene in both the root and shoot, whereas $A B F 3$ is only correlated in the root. The $\mathrm{ABA}$, cytokinin, and circadian rhythm had a strong correlation with $A B A$ signaling genes (interaction, in particular $A B F 2, A B F 3$, SnRK2, cytokinin response factor (CRF4) Arabidopsis histidine phosphotransferase (AHP4), gigantea (GI), constans-like (COL3), and phytochrome interacting factor 4 (Fig. 5a). In WD shoot, strong correlations between $\mathrm{ABA}$ and cytokinin signaling genes also showed similar correlation with the circadian rhythm genes: $A B F 2$, histidine kinase 1 ( $A H K 3$ ), and Arabidopsis type b cytokinin response regulators (ARR1) signaling genes were correlated with $G I$ and timing of cab expression 1 protein (TOC1) (Fig. 5b). A total gene list for each network is included in the Additional file 7: Table S5a-d.

\section{Aquaporin and ROS scavenging genes had distinctly different expression patterns in water deficit root and shoot}

Examination of the VitisNet pathways significantly enriched in WD root or WD shoot indicated that several gene families previously noted to be involved in water deficit responses were not only differentially expressed relative to their control but showed different response profiles in root and shoot. Major differences were observed in aquaporin and reactive oxygen species scavenging (ROS) DEGs. It is noteworthy that there were only two up-regulated aquaporins in WD roots (VIT_08s0040g018 90, VIT_14S0108g00700) and 16 aquaporin DEGs that were all down-regulated in WD shoot (Additional file 3: Table S3, Additional file 4: Table S4).

A greater number of ROS scavenging related genes were differentially expressed in the WD shoot than in the WD root relative to their respective $\mathrm{C}$ (Fig. 6, Additional file 8: Table S6). In WD shoot, six of the 36 genes responsible for synthesizing superoxide dismutase $(S O D)$ were downregulated and three were up-regulated relative to $C$ shoot. Only two SOD genes were up-regulated in WD root and one (VIT_14s0060g00120) was down-regulated in common with WD shoot. Many more genes in the glutathione cycle were up-regulated in the WD shoot (22) than in the WD root (11). Four glutathione reductase $(G R)$ genes were differentially expressed in WD shoot, with one of the down-regulated in common with WD root. Genes encoding glutathione peroxidases (GPX: VIT_02s0025g03600, VIT_04s0008g06780) were up-regulated in WD shoot, with one in common with WD root. Two ascorbate peroxidase $(A P X)$ genes were up-regulated in WD shoot and none were differentially expressed in WD root. In contrast, three CATALASE (CAT) genes were down-regulated in WD root and one up-regulated in WD shoot. The phenylpropanoid pathway is a source of other potential antioxidant compounds (lignins, stilbenes, flavonoids, and anthocyanins). The WD shoots had a greater number of up-regulated phenylpropanoid biosynthesis DEGs than WD roots. Some genes in the flavonoid synthesis pathway were up-regulated, but more were down-regulated including stilbene synthases.

\section{Different transcription factors profiles were associated with WD in root and shoot}

There were 62 transcription factors up-regulated and ten down-regulated in common between WD root and WD shoot, and eight that were differentially regulated (Additional file 3: Table S3, Additional file 4: Table S4). 

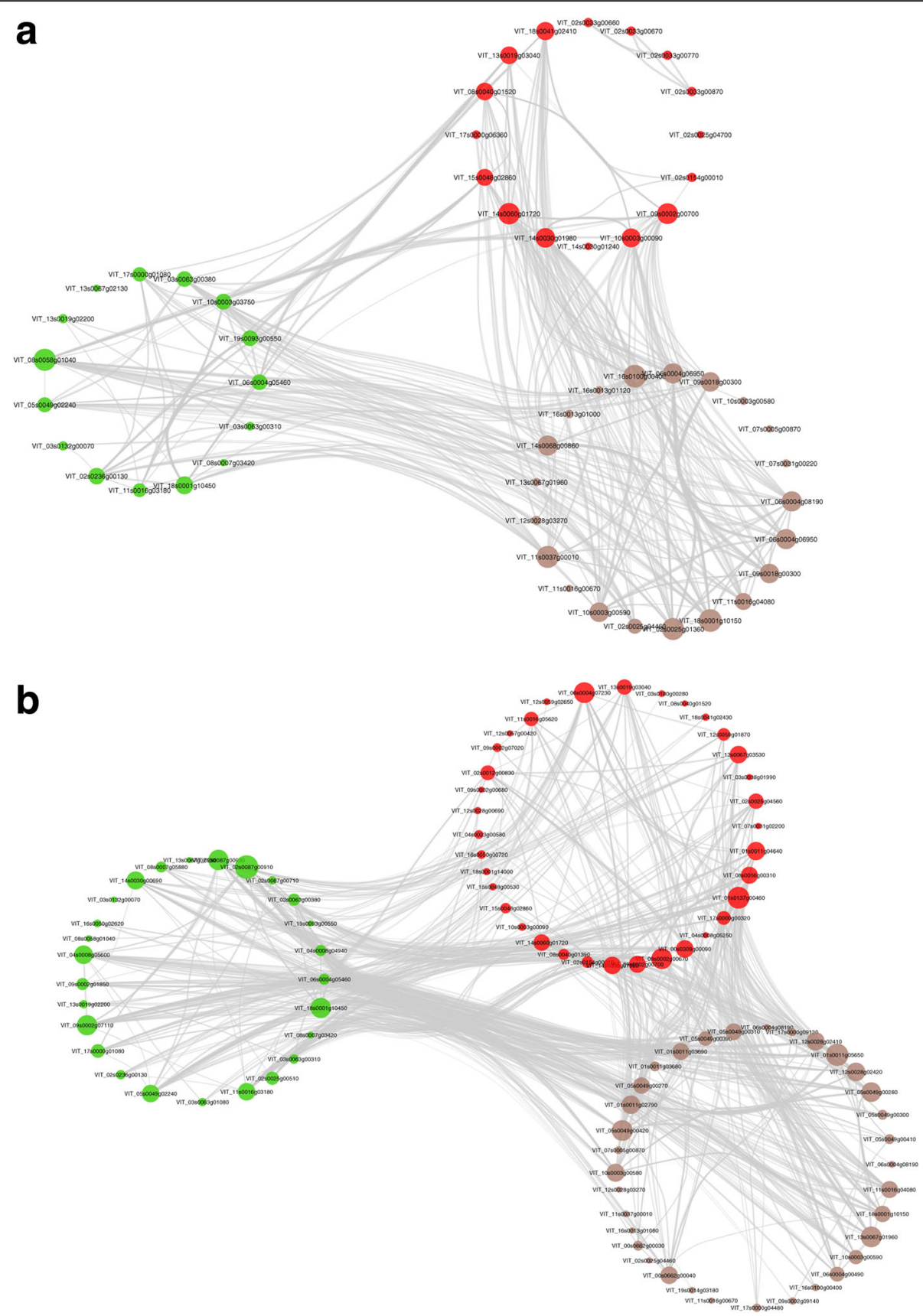

Fig. 4 Root and shoot ABA, auxin, and ethylene signaling networks. a root network; $\mathbf{b}$ shoot network. The differentially expressed gene correlation matrix was modeled using the graph from adjacency matrix function in igraph $\mathrm{R}$ package ( $\mathrm{ABA}=$ light green, auxin $=$ red, ethylene $=$ grey-brown). Network gene list and annotation can be found in Additional file 7: Table S5a, b

A large transcriptional regulatory activity potential was indicated in the WD shoot. A large number of transcription factor DEGs (220 up-regulated, 264 down-regulated) were expressed only in the WD shoot; in contrast, 42 DEGs were expressed only in WD root (Additional file 3: Table S3, Additional file 4: Table S4). The transcription factors (NAC, C2C2-DOF, C2C2-YABBY, G2-like, other ZF-AN1, and GNAT), which have been associated with drought tolerance in grapevine [14, 18] were significantly enriched in WD root (Additional file 3: Table S3). A greater number of NAC (Fig. 7a) DEGs were up-regulated in WD shoot (17) than WD root (9). There were 8 NAC DEGs in common between the WD organs (Additional file 3: Table S3, Additional file 4: Table S4). The majority of the WRKY DEGs in the WD shoot were up-regulated (Fig. 7b) and there 


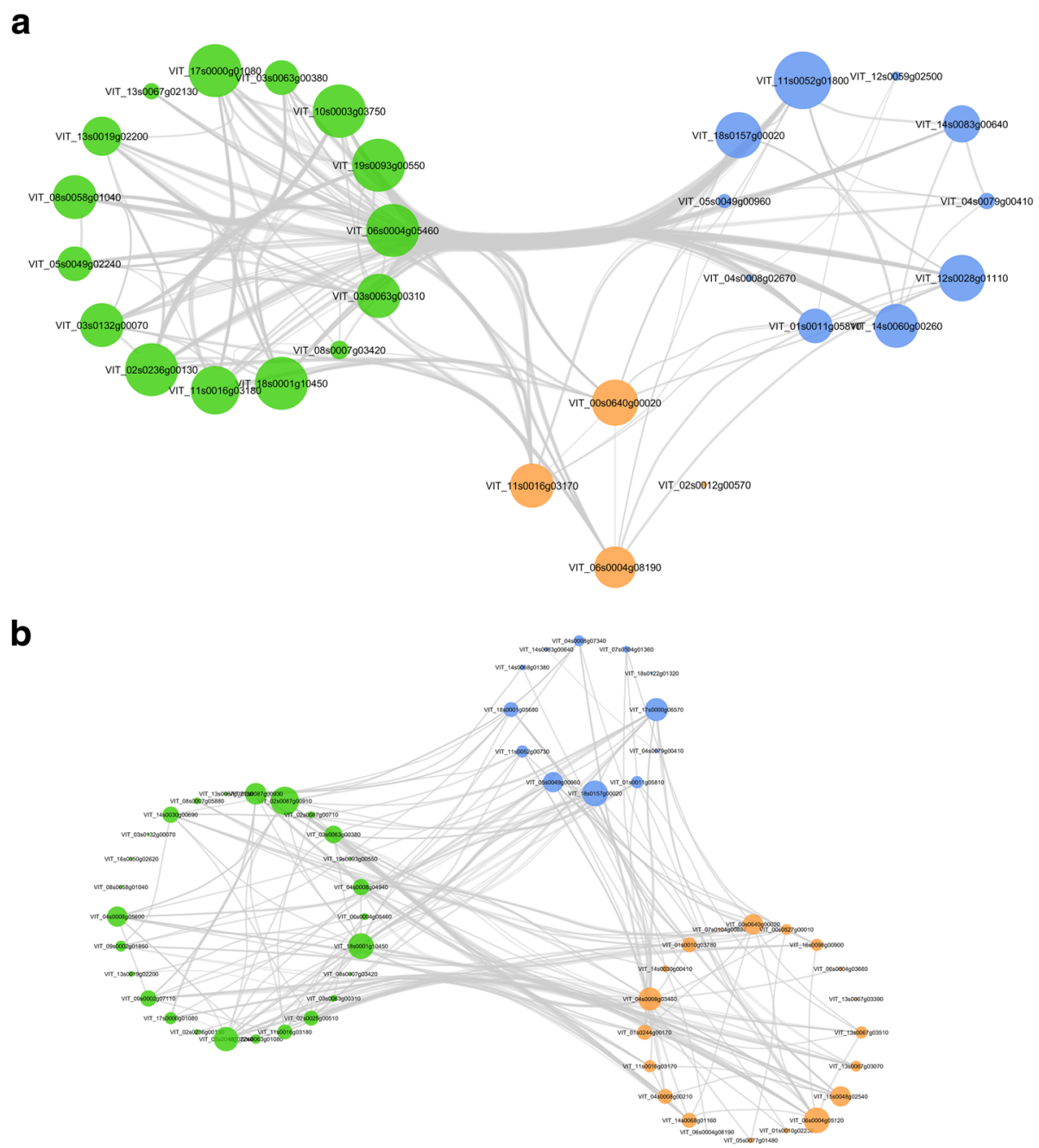

Fig. 5 Root and shoot ABA, cytokinin, and circadian rhythm signaling network. a root network; b shoot network. The differentially expressed gene correlation matrix was modeled using the graph from adjacency matrix function in igraph $\mathrm{R}$ package $(\mathrm{ABA}=$ light green, cytokinin = blue, circadian rhythm = orange). Network gene list are found in Additional file 7: Table S5c, d

was only one up-regulated WRKY transcription factor DEG in WD root (WRKY23, VIT_07s0005g01710). A larger number of $M Y B$ s, which target genes that are involved in the biosynthesis of phytohormones and cell walls, were differentially expressed in WD shoot $(71,32)$ in contrast to WD root $(18,9)$ (total, up-regulated respectively) (Fig. 8a). It should be noted that 15 of these were DEGs in both WD organs. A similar pattern was observed in the basic helix loop helix $(b H L H)$, transcriptional activators in ABA signaling; however, few were in common with bHLHs DEGs in WD root (Fig. 8b). There were few dehydration responsive element binding (DREB) transcription factors expressed in WD shoot, and only one in WD root. The bZIP transcription factors that are regulators for the ABA mediated abiotic stress signaling pathways, were up-regulated in WD root and up- and down-regulated in WD shoot.

\section{Discussion}

This study provided a physiological and genome-wide transcriptomic analyses of water deficit responses of shoots and root in $V$. riparia, a species commonly used as a rootstock and in breeding rootstock and scion cultivars. Shoot elongation, photosynthetic and stomatal activity, water status, and morphological characters were observed to target the time point for transcriptomic analysis. $V$. riparia had decreased shoot elongation after 10 d of water deficit, similar to other studies of potted grapevines [44-46]. After $14 \mathrm{~d}$ of water deficit, the physiological status of the grapevine characterized by $\Psi_{\text {stem, }} \mathrm{P}_{\mathrm{n}}, \mathrm{SC}$, and WC was significantly impacted in WD grapevines relative to the control grapevines. Leaf shed, cupping, and wilting were observed at $14 \mathrm{~d}$ of WD. Leaf shed has been shown to reduce water loss during periods of stress in grapevine [47]. In addition, shedding 


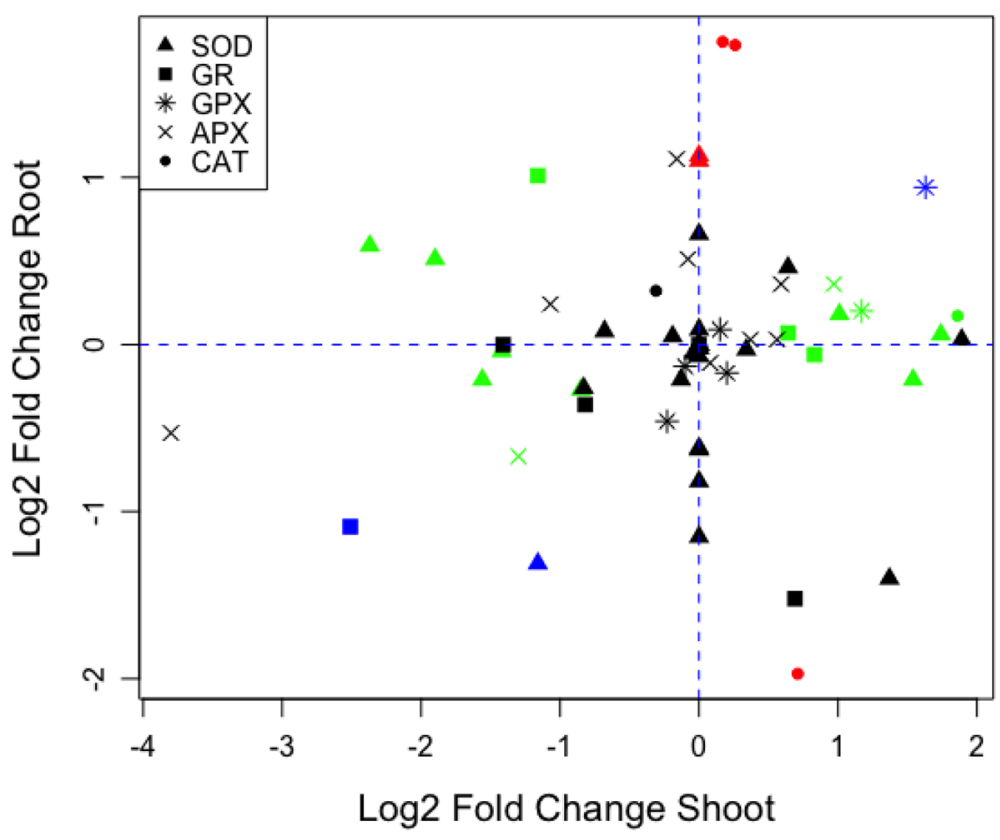

Fig. 6 Scatter plot of fold change of genes associated with reactive oxygen species (ROS) scavenging enzymes. ROS scavenging gene families are identified in the legend at top right and differentially expressed (q-value $<0.05)$. Superoxide dismutase $(S O D$, triangle), glutathione reductase $(G R$, square), glutathione peroxidase (GPX, star), ascorbate peroxidase (APX, $x$ ) and catalase (CAT, circle) related genes are color coded for shoot (green) and root (red). Genes right and left of the vertical dash line represent up- and down- regulated, respectively in water deficit (WD) relative to well-watered control (C) shoot (green). Genes above and below the horizontal line represent up- and down- regulated respectively in WD root relative to $C$ root (red). Markers for gene family members differentially expressed in both root and shoot are blue and markers for genes not differentially expressed are black. A complete list of ROS related genes are found in Additional file 8: Table S6

older leaves allows reallocation of the carbon, nitrogen, and other nutrient resources to support growth in the stem or younger leaves in grapevine and other woody species $[20,48,49]$. Changes were observed visually in the root balls; roots became brown and less flexible, and there were fewer roots visible in WD compared to $\mathrm{C}$ grapevines. These characteristics indicate that both root and shoot were adjusting to the water deficit treatment. In this study; however, there were three times as many DEGs in WD shoot than in WD root in strong contrast to a previous WD study in grapevine rootstocks [18]. The difference between the present study and the rootstock study [18] may be due to differences in genotype, WD sensitivity or differences in the length of the water deficit treatment [50].

\section{Energy related pathways were enriched in WD root}

Water deficits in plants cause stomatal closure in the leaves, inhibition of photosynthetic activity and reduction in carbon fixation [51]. Water stress accompanied with heat enhances respiration and methane $\left(\mathrm{CH}_{4}\right)$ emission [52]. In V. riparia, photosynthesis and stomatal conductance decreased in the WD treatment. In contrast, VitisNet photosynthesis molecular pathways were positively enriched and genes in both photosystem I and II were upregulated in WD root and WD shoot. A similar decrease in net photosynthesis and up-regulation in photosynthesis related genes and proteins was observed in water-stressed rootstock and scion varieties [13, 27, 53].

Plants alter carbohydrate metabolism equilibrium by accumulating a large amount of water-soluble carbohydrates. In rice, carbohydrate metabolism pathways (fructose and mannose; starch and sucrose metabolism; amino sugars and nucleotide sugars and galactose) are significantly enriched during drought [54]. In contrast, amino sugars and nucleotide sugar metabolism pathways were negatively enriched in WD shoot and WD root in this study. In the woody Jatropha curcas, starch, sucrose, and galactose pathways are enriched in response to drought and have the greatest number of DEGs in common to both root and leaves under drought conditions [48]. Similarly, in WD grapevine, galactose metabolism was positively enriched in both WD root and WD shoot. Similar increases in galactose metabolism DEGs and proteins are observed in leaves of rootstocks and Cabernet Sauvignon [14, 27]. Galactose metabolism is important in drought tolerance mechanisms and may provide antioxidant activities $[55,56]$. This may also indicate carbohydrate accumulation in grapevine in response to growth suppression under water deficit or down-regulation of sugar transporters [57]. 


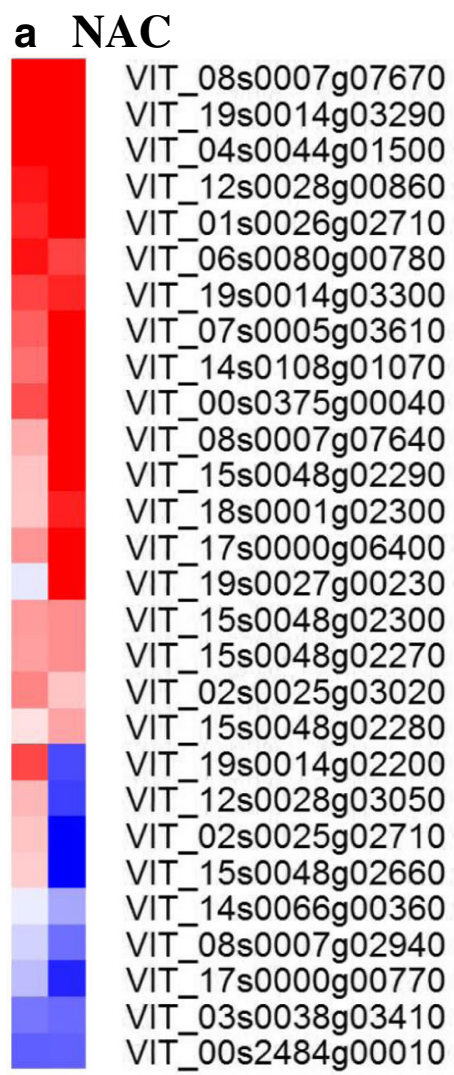

\begin{tabular}{l}
$t \overrightarrow{0}$ \\
\hdashline$\frac{0}{\omega}$
\end{tabular}

\section{b WRKY}

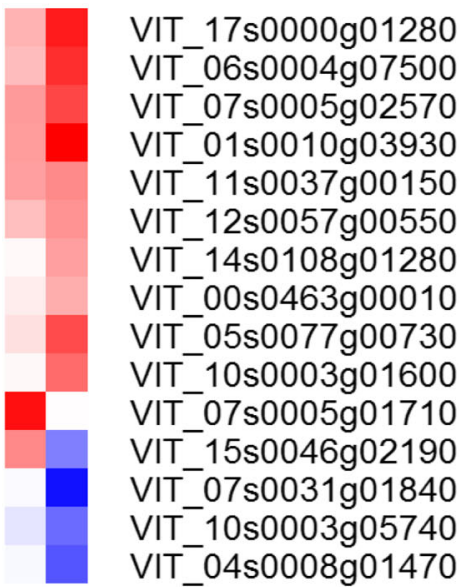

$\overrightarrow{0}$
$\ddot{\varnothing} \frac{⿱}{\omega}$

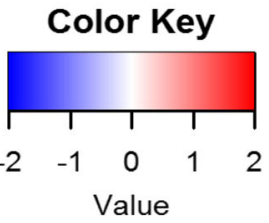

Fig. 7 NAC and WRKY transcription factor differential gene expression (DEG) profile in water deficit (WD) root and shoot tissue. a NAC transcription factor DEG. b WRKY transcription factor DEG. The red and blue colors represent up- and down-regulation of the gene expression in water deficit tissue relative to their respect to control tissue in root or shoot. Expression values are expressed fold change WD/C $\left(\log _{2}\right)$

\section{Cell structure and function pathways were enriched in} WD shoot

Actin cytoskeleton mediates cell motility and shape changes during the cell-division cycle and in response to extracellular stimuli [58]. Osmotic stress causes rapid and reversible changes in actin filament organization [59]. In grapevine, the actin cytoskeleton pathway was negatively enriched in WD shoot most likely due to decreased growth under water deficit.

Changes in lipid composition help maintain membrane integrity and preserve cell compartmentation in plants during drought. Leaf lipid content in Arabidopsis thaliana decreases progressively under drought [60]. In grapevine, drought tolerant cultivars show an increase in total lipid content in the leaves, in contrast to the drought sensitive cultivars [61]. In $V$. riparia, the fatty acid biosynthesis pathway was negatively enriched in WD root and WD shoot. In contrast, the glycerolipid pathway was upregulated in both the WD organs. This coupled with the up-regulation of the galactose pathway indicates active maintenance of the photosynthetic membranes under water deficit conditions [62].

The autophagy pathway was enriched in both the WD root and WD shoot in $V$. riparia. Autophagy plays a role in protein quality control by targeted degradation of misfolded and damaged proteins induced during biotic and abiotic stresses [63]. Autophagy genes are differentially regulated in roots and shoots of drought-stressed pepper, with a greater up-regulation in roots and stems than in leaves [64]. The heat shock transcription factor A1a (HsfA1a) regulates autophagy related genes and overexpression of $H s f A 1$ a promotes non-ABA dependent drought tolerance and autophagosome formation in tomato [65]. In WD shoot several autophagy genes were up-regulated and phagosome genes were both up- and down-regulated. Several heat shock transcription factors were up-regulated in the WD shoot; however, an ortholog was not found for HsfAla. Further comparative exploration of these genes in drought tolerant and sensitive cultivars may provide greater understanding in grapevine. 


\section{a MYB}

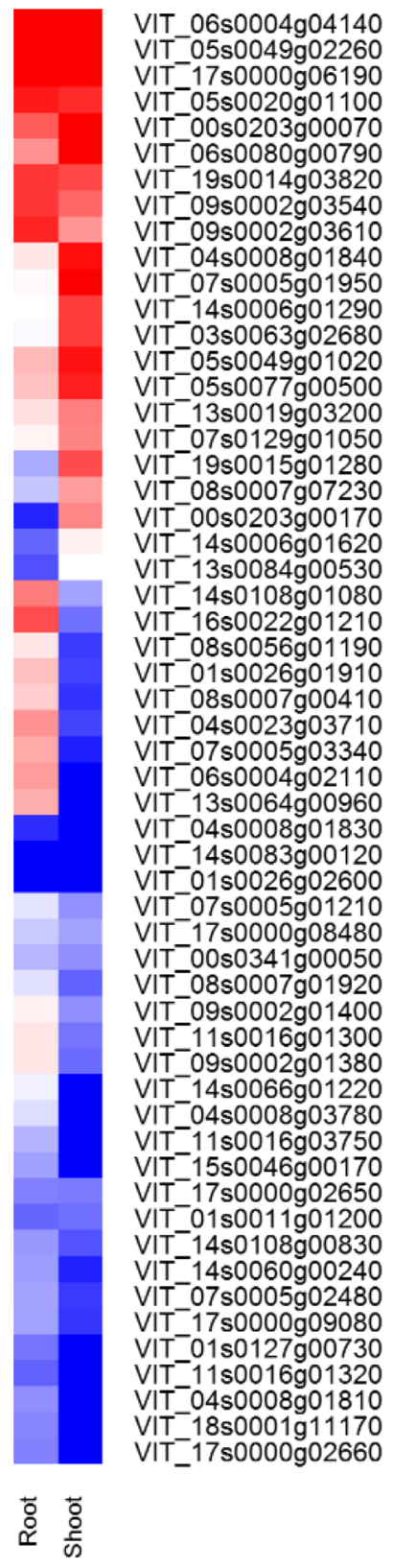

\section{b bHLH}
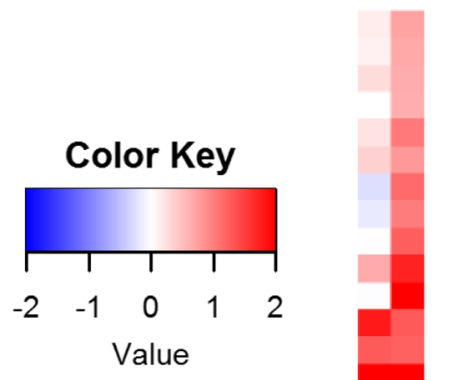

VIT_01s0244g00130

VIT-02s0012g01320

VIT-11s0037g00040

VIT-12s0059g02650

VIT-14s0060g01010

VIT-18s0041g00690

VIT-08s0007g07810

VIT-12s0028g03550

VIT-13s0064g01290

VIT-05s0020g04620

VIT-17s0000g08150

VIT-07s0031g00450

VIT-15s0021g02690

VIT-18s0001g07410

VIT-17s0000g00330

VIT-17s0000g03580

VIT-14s0108g00480

VIT-11s0016g00380

VIT-12s0028g01110

VIT $1450060 \mathrm{~g} 00260$

VIT-14s0068g01580

VIT-14s0006g02850

VIT-08s0058g00110

VIT-03s0038g04760

VIT-05s0020g04780

VIT-13s0073g00400

VIT-06s0061g00720

VIT-08s0058g00960

VIT-03s0088g01240

VIT-18s0001g06650

VIT-07s0191g00240

VIT-18s0001g08040

VIT-02s0025g03450

VIT-15s0046g02560

VIT-O0s1312g00010

VIT-12s0034g02150

VIT-05s0029g00390

VIT-01s0011 g02940

VIT-08s0007g00940

VIT-08s0040g01240

VIT-18s0001g07200

VIT-02s0012g01450

VIT-18s0001g08600

VIT-09s0002g04120

VIT-18s0001g12970

VIT-18s0001g07210

VIT-15s0048g02510

VIT-05s0077g00750

VIT-01s0026g02030

VIT-02s0025g02310

VIT-08s0007g07870

VIT-04s0023g01930

VIT-01s0010g02070

VIT-17s0000g03550

VIT-01s0026g01140

VIT-17s0000g04790

VIT-14s0108g00420

VIT-07s0151g00450

VIT-17s0000g00430

VIT_14s0128g00110

客高

Fig. $8 M Y B$ and $b H L H$ transcription differential gene expression (DEG) profile in water deficit (WD) root and shoot tissue. a MYB transcription factor DEG. $\mathbf{b} b H L H$ transcription factor DEG. The red and blue colors represent up- and down-regulation of the gene expression in water deficit tissue relative to their respect to control tissue in root or shoot. Expression values are expressed fold change WD/C ( $\left.\log _{2}\right)$

\section{ROS scavenging gene enrichment in WD shoot}

In the leaves, stomata closure in response to low shoot water potentials and soil moisture content contributes to reduced photosynthesis and increased respiration [24, 51, 66]. Under low $\mathrm{CO}_{2}$ and excess light, reactive oxygen species are formed due to partial reduction or activated derivatives of molecular oxygen from the electron transport activities of chloroplasts, mitochondria, and peroxisomes $[67,68]$. Prolonged water stress leads to overproduction of reactive oxygen species that exceeds cellular quenching mechanisms thus disrupting cellular homeostasis [69]. This results in oxidation of proteins, peroxidation 
of lipids, damage to nucleic acids, inhibition of enzymes, activation of programmed cell death pathway and ultimately cell death $[69,70]$. ROS scavenging enzymes are early stress signaling messengers often associated with drought-induced ABA synthesis in water stressed plants $[13,15]$. The present study showed a greater number of ROS scavenging genes DEGs particularly in WD shoot, which was similar to increases noted in $V$. vinifera scion and rootstock cultivar leaves during drought stress and may be related to the electron transport activity in leaves $[13,27,71]$.

\section{ABA biosynthesis and ABA signaling had common water deficit transcriptomic responses in roots and shoots}

Grapevine leaf dehydration and potted and field water deficit studies indicate a strong role for $\mathrm{ABA}$ and other hormone signaling in grapevine acclimation to water stress $[13,14,17,18]$. Prior to ABA synthesis, carotenoid biosynthesis initiates with the synthesis of the isopentenyl pyrophosphate (IPP), and gradually feeds into the ABA biosynthesis pathway [27]. Zeaxanthin epoxidase ( $A B A 1)$ catalyzes an early step in the cascade towards ABA biosynthesis [72]. In $V$. riparia, $A B A 1$ expression was up-regulated in WD root but down-regulated in WD shoot. Increased activity of $A B A 1$ in WD root was also observed in tomato and Arabidopsis under water stress [73, 74]. In addition, the $V D E$ genes were also up-regulated in WD root but down-regulated in WD shoot. In the ABA metabolic pathway, the first committed step in ABA biosynthesis is initiated by 9-cis-epoxycarotenoid dioxygenase (NCED), which converts violaxanthin or neoxanthin to xanthoxin. Two of the three NCED genes were up-regulated in WD root, but only one in WD shoot. This result was in agreement with results in rootstocks and scions $[17,18]$ that show NCED transcripts increase in both shoot and root in response to water deficit; thus indicating a strong role for root $\mathrm{ABA}$ signaling to the shoot.

ABA levels can be decreased by ABA catabolism, inactivation, or negative regulation. The $A B A$ 8'-hydroxylase enzyme catabolizes $A B A$ into 8'- hydroxy $A B A$, which non-enzymatically converts to phaseic acid before reducing to dihydroxyphaseic acid. ABA, in the presence of an ABA-glucosyltransferase enzyme, is metabolized into inactive ABA-glucose ester (ABA-GE). Cleavage of glucose from $A B A$-glucose ester by $\beta$-glucosidases converts ABA-GE back to ABA. Although water stress acts as a signal to transport ABA-GE stored in the vacuole or apoplastic space to the endoplasmic reticulum, its involvement in root-to-shoot signaling is yet to be revealed $[29,75,76]$. Several studies report an increase of UDP-glycosyltransferase under stress conditions [77, 78]. ABA-GE concentration increases significantly in WD Chardonnay berries but not in Cabernet Sauvignon [29]. In this study, several UDP-glycosyltransferase genes were up-regulated in the
WD shoot, but not in the WD root. Further studies are needed to get a complete picture of how ABA levels are controlled in grapevine.

ABA signaling genes involved in the water stress response include $P Y R / P Y L / R C A R, P P 2 C$ (negative regulator) and SnRK2 (positive regulator) [28]. The ABA receptor $\mathrm{PYR} / \mathrm{PYL} / \mathrm{RCAR}$ family controls $\mathrm{ABA}$ signaling by inhibition of $P P 2 C$ activity [79-82]. Major inhibition of $P P 2 C$ occurs in the presence of ABA; however, the ABA receptors (RCAR/PYR1/PYL) are known to interact with PP2Cs in a complex manner [83, 84]. SnRK2, when activated by $\mathrm{ABA}$, phosphorylates ABA-responsive element binding factors $(A B F S)$. In addition, target of rapamycin (TOR) kinase, a central regulator of plant metabolism and growth, phosphorylates PYR/PYL/RCAR receptors in unstressed conditions, inactivating the core $\mathrm{ABA}$ signaling pathway [85]. Under stressed conditions, ABA stimulated SnRKs, phosphorylate the TOR complex component, RAPTOR, providing a mechanism for altering cell metabolism and growth. In $V$. vinifera and hybrid rootstocks, high constitutive expression levels of $P Y R / P Y L / R C A R s$ and $S n R K s$ are found indicating that the transcript abundance of ABA receptors was not a key component contributing to genotypic differences in water deficit response [17]. However, in a grapevine leaf dehydration study [14], differences in SnRK 2.6 (OST1) are found in gene expression profiles over time, with $V$. riparia having the lowest response. In the present V. riparia study, SnRK2.2 was up-regulated in both WD root and WD shoot, indicating a difference between isolated leaf dehydration and whole plant water deficit signaling.

\section{Cross-talk between ethylene, cytokinin, circadian rhythm and ABA signaling pathways}

The expression profiles of the ABA, ethylene, cytokinin, and circadian rhythm signaling in response to water deficit in the shoot and root suggest WD induced hormone cross-talk [14, 86]. Coexpression networks constructed for leaf dehydration responses indicate key regulatory roles for ABA and ethylene signaling [14]. Similarly, networks constructed for hormone signaling in $V$. riparia WD roots and WD shoots had strong correlations of ABA with ethylene signaling with both ERF and AP2 domain-containing transcription factor/ERF up regulated in response to water deficit. In contrast, Auxin signaling did not have a strong correlation in $V$. riparia with ABA signaling, but auxin and ethylene signaling were more strongly related in both root and shoot under WD. The strong correlation of the ABA with the ethylene network found in WD root and WD shoot in the present study supports the role of cross-talk between ABA signaling and ethylene signaling and its proposed mediation by $A B F 2$ and $A B I 5$ identified in leaf dehydration studies [14]. It is of particular interest that although $A B F 2$ was 
strongly correlated in leaves and roots in the present study, $A B F 3$ was only correlated in the root, suggesting a root specific role for $A B F 3$.

The potential for cross-talk between ABA and cytokinin is strong, as type-a arabidopsis response regulators (ARR4, ARR5 ARR6) interact with and regulate expression levels of the ABA signaling gene, abscisic acid insensitive $5(A B I 5)$, in addition to regulating cytokinin signaling [87]. Furthermore, three cytokinin receptor histidine kinases ( $A H K 2, A H K 3$, and $A H K 4)$ are negative regulators of ABA signaling [88]. A bidirectional cross-talk is noted for ABA and circadian rhythm signaling $[89,90]$. In $V$. riparia WD roots, $A B I 5-1$ is up-regulated and there are no $A R R$ DEGs (negative regulator). In the WD shoot, $A R R 4$ and ARR5 (VIT_01s0026g00940 and VIT_17s000g07580) are significantly down regulated and $A B I 5$ is up-regulated. Hopper et al. [14] identified $A B F 2$ and $A B I 5-1$ as key ABA signaling hubs whose expression correlated with the drought tolerance of the leaves of three grapevine species. The up-regulation of $A B F 2$ and $A B I 5-1$ in WD root and WD shoot in the current study support the proposed major role of these signaling genes in water deficit signaling in grapevine [14].

Circadian clock gating regulates ABA signaling networks [90]. MYB96 transcription factor contributes to the gating of abscisic acid responses by binding to timing of cab expression 1 (TOC1) [76]. TOC1 binds to the promoter of ABA related gene $(A B A R)$ and TOC1 is induced by ABA [91, 92]. Constitutive expression of clock-associated pseudo-response regulator $(P R R)$ genes improves drought tolerance in Arabidopsis [93], supporting the hypothesis of the involvement of cytokinin signaling in response to water deficit. The common expression profile characteristics of the ABA, cytokinin, and circadian rhythm signaling in response to WD in this study, indicated that cross-talk between the hormone signaling and clock associated genes may play a role in water deficit response. The circadian clock has been strongly implicated in growth and development synchronization and changes that impact development of organs could be expected to show circadian perturbations. In particular, it has been noted that the circadian clock rephrases during lateral root initiation indicating a role of the circadian clock in adapting growth cycling to environmental conditions [94].

In this study, the circadian rhythm and ABA and cytokinin signaling showed strong correlations with COL3 and TOC1 expression in the WD root and WD shoot, respectively. In addition, GI, which is increasingly implicated in abiotic stress tolerance [86], was identified as a contributing member of both the WD root and shoot ABA, cytokinin and circadian rhythm cross-talk networks. While $\mathrm{ABA}$ and circadian rhythm gene interactions have been noted in $\mathrm{ABA}$ gating in the leaves in response to water deficit [90] or root growth in relation to carbohydrate availability [95], interactions of ABA, cytokinins, and circadian rhythms have not been previously reported in root in response to water deficits in grapevine. These results suggest a strong role for circadian clock mechanisms in grapevine root acclimation to soil water changes.

\section{Water deficit-responsive transcription factor profiles are different in WD root than WD shoot}

Transcription factors greatly influence the plant's responses to water deficits. The responses vary with genotype and organ, indicating different signaling networks are involved in the complex plant responses to water deficit. ABA-independent signaling pathways like NAC transcription factors are known to be involved in drought stress responses and modulate downstream early response to dehydration1 gene transcription [96, 97]. The NAC transcription factors are differentially expressed in grapevine in response to abiotic stress [98]. NAC transcription factors expression change in leaves in response to water deficit [26]. A large number of NACs are differentially expressed in two rootstocks (M4 and 101.14) with 14 differentially expressed in both roots and leaves during water deficit treatments [18]. There were fewer differentially expressed in $V$. riparia in this study; however, eight of the genes that were DEGs in WD shoot and WD root are found in the Corso et al. [18] rootstock study in response to WD. Although there were more differentially expressed $N A C$ transcription factor genes in the $V$. riparia WD shoot, in contrast, to the NACs in M4 and 101.14 rootstock leaves. This suggests that while up-regulation of $N A C$ transcription factors has been associated with increased drought tolerance, careful review of each $N A C$ must be conducted by organ to identify this transcription factor's role in drought tolerance. A V. amurensis NACO29 (VIT_01s0026g02710) improves drought tolerance, modulates jasmonic acid synthesis, and enhances ROS scavenging enzymes in transgenic Arabidopsis [99]. In the present study, seven of the $V$. riparia $N A C$ were up-regulated in both root and shoot in response to WD, suggesting a specific role for these particular NAC transcription factors in water deficit tolerance that may be related to ROS scavenging enzyme regulation.

$M Y B$, a large transcription factor family, triggers the expression of drought- and ABA- induced genes to improve drought tolerance [100-102]. Ectopic expression of OsMYB4 transcription factor is associated with physiological and biochemical adaptation to drought stress in apples [103]. In V. riparia, flavonoid biosynthesis was generally up-regulated in WD shoot in contrast to WD root. There were more differentially expressed $M Y B$ transcription factors in WD shoot than WD root in $V$. riparia. This was in contrast with M4 
and 101.14 root, which had more MYBs differentially expressed in the roots than in leaves under water deficit [18]. There were more MYBs differentially expressed in $V$. riparia than M4 and 101.14; however, the MYBs that were common in the WD treated shoots of each of the three genotypes were up-regulated, suggesting a regulatory role for $M Y B$ in water deficit shoot.

\section{Conclusions}

Mature leaf abscission, morphological root changes, and shoot growth cessation were found during the water deficit treatments and indicated that $V$. riparia may be reallocating its limited resources to maintain the apical portion of the grapevine shoot. An overall decrease in photosynthesis and an increase in dry matter content of roots demonstrated that $V$. riparia reallocated resources to sustain potential changes in the root that contributed to water maintenance in the early stages of water deficit. RNAseq transcriptome profiling of $V$. riparia revealed significant impacts of WD in roots and shoots. The larger number of DEGs and enriched VitisNet pathways found in WD shoot indicated that the shoot had a greater response to water deficit than the grapevine root. There were a large number of DEGs in response to WD. In addition, 10 VitisNet pathways were enriched in common with both WD root and WD shoot: notably, ABA biosynthesis, cytokinin signaling, NAC transcription factors and galactose metabolism. The transcript abundance of genes for enzymes involved in carotenoid and ABA biosynthesis ( $A B A 1, V D E$, and NCED3) increased in both WD root and WD shoot relative to their respective well-watered controls. There were more ROS DEGs in WD shoot than WD root, indicative of greater potential ROS scavenging action, most likely related to the photosynthetic capacity of the shoot. Impact on water movement capacity, as evidenced by aquaporin gene expression, presented two aquaporins that were up-regulated in the roots. In contrast, all aquaporin DEGs in the shoots were down-regulated. This suggested that aquaporins contributed to water uptake in the root and limited water loss in the shoot. Specific members of the NAC transcription factor family had similar responses and identified a common signaling role in both WD root and WD shoot. Key genes common in WD root and WD shoot were identified from the interacting hormone expression networks between ABA, ethylene, cytokinin, and circadian rhythm genes. These expression patterns suggested potential genes, as well as organ specific genes, to be explored in future regulatory time course studies. The combined shoot and root responses indicated the involvement of complex signaling networks in water deficit responses in grapevine roots as have been previously described for WD shoots [14]. In addition, the root expression data supported an ABA, cytokinin, and circadian rhythm signaling network in grapevine roots in response to water deficit.

\section{Additional files}

\begin{abstract}
Additional file 1: Table S1. RT-PCR candidate gene information. Candidate gene name, Unique ID, forward (F) and reverse (R) primer sequences, optimal primer concentrations (nM), amplification efficiency (\%), and correlation coefficient for fold change of RT-PCR and RNAseq expression values (R2) of each gene in root and shoot. (XLSX $10 \mathrm{~kb}$ )
\end{abstract}

Additional file 2: Table S2. Root and shoot water and dry matter content. Mean \% Water content (WC), hydration value (HV) and dry matter content (DMC) of grapevine tissues. Two-way ANOVA significance noted by: $y=$ main effect of Day of Treatment; $z$ = main effect of Water Status Treatment; $y z=$ significant main effects of water status and day at $p \leq 0.05$. (XLSX $9 \mathrm{~kb}$ )

Additional file 3: Table S3. Differentially expressed genes in water defict root (WD root) and well-watered root (C root). Values are normalized RPKM for each treatment, fold change (FC) and test statistic and q-value. Genes highlighted in gray were up-regulated in WD root relative to $C$ root. Genes in bold were differentially express in common in WD root and WD shoot relative to their respective $C$ tissue. (XLSX $176 \mathrm{~kb}$ )

Additional file 4: Table S4. Differentially expressed genes in water defict shoot (WD shoot) and well-watered shoot ( $C$ shoot). Values are normalized RPKM for each treatment, fold change (FC) and test statistic and q-value. Genes highlighted in gray were up-regulated in WD shoot relative to $C$ shoot. Genes in bold were differentially express in common in WD root and WD shoot relative to their respective C tissue. (XLSX $673 \mathrm{~kb}$ )

Additional file 5: Figure S1. Shoot and root DEG in common during water deficit. Values are fold change (FC, log2) of water deficit root and shoot relative to their respective control. (PPTX 61 kb)

Additional file 6: Figure S2. Expression fold change for ABA metabolic and signaling genes in water deficit (WD) roots and shoot tips. All values were measured using real-time PCR and ratio of water deficit /control (WD/C)). Gene ids are ABA1 (VIT_07s0031g00620), NCED3 (VIT_19s0093g00550), PP2CA (VIT_13s0019g02200), CYP707A3 (VIT_02s0087g00710), ABA3 (VIT_19s0027g01090), and AB/1 (VIT_11s0016g03180). Root = solid bar, Shoot = striped bars; values with asterix denote genes differentially expressed between tissues, with a $p$-value $<0.05$ noted above bar $(n=3)$. (PPTX $43 \mathrm{~kb}$ )

Additional file 7: Table S5. Hormone signaling networks. A. ABA, auxin, and ethylene network for $V$. riparia root. B. ABA, auxin, and ethylene network for $V$. riparia shoot. 5C. ABA, cytokinin, and circadian rhythm network for $V$. riparia root. D. ABA, cytokinin, and circadian rhythm network for $V$. riparia shoot. (XLSX $16 \mathrm{~kb}$ )

Additional file 8: Table S6. RPKM expression fold change (FC log2) of reactive oxygen species scavenging enzymes in water deficit (WD) relative to well-watered organ (C) for root and shoot. Enzymes are noted as superoxide dismutase (SOD), glutathione reductase (GR), glutathione peroxidase (GPX), catalase (CAT), and ascorbate peroxidase (APX). Differentially expressed genes (DEG) at q-value $<0.05$ are in bold lettering and highlighted according to organ in which DEG occured; root (gray), shoot (green), and both WD organs relative to their respective $C$ (yellow). (XLSX $10 \mathrm{~kb})$

\section{Abbreviations}

ABA: Abscisic acid; C: Control; DEGs: Differentially expressed genes; DW: Dry weight; FDR: False discovery rate; FW: Fresh weight; Pn: Net photosynthesis; SC: Stomatal conductance; WD: Water deficit; $\Psi_{\text {stem: }}$ Stem water potential

\section{Acknowledgements}

Not applicable

\section{Funding}

We acknowledge support from National Science Foundation (NSF) Plant Genome Research Program award DBI\#0604755 and \#0741876, NSF/EPSCoR 
Cooperative Agreement \#IIA-1355423, BioSNTR which is funded in part by the South Dakota Research and Innovation Center and USDA National Institute of Food and Agriculture, Hatch project SD0OH449-12 that supported this research. VK was partly supported by the National Institutes of Health U54MD007584, U54MD007601 and P20GM103466. Any opinions, findings, and conclusions or recommendations expressed in this material are those of the author(s) and do not necessarily reflect the views of the National Science Foundation, National Institutes of Health or U.S.

Department of Agriculture.

\section{Availability of data and materials}

The datasets generated for this study can be found in the NCBI sequence read archive under accession\#GSE109065, SRA SRP130959.

\section{Authors' contributions}

$A F, K V, V K$, and GRC developed the experimental design. KV, VK, JX, and AF conducted experiments and analyzed data. VK, KV, AF, PS, JX, QM, and GRC developed manuscript. All authors read and approved the final manuscript.

\section{Ethics approval and consent to participate}

Not applicable.

\section{Consent for publication}

Not applicable.

\section{Competing interests}

The authors declare that the research was conducted in the absence of any commercial or financial relationships that could be construed as a potentia conflict of interest. GRC is a member of the editorial board (Section Editor) for BMCPlantBiology.

\section{Publisher's Note}

Springer Nature remains neutral with regard to jurisdictional claims in published maps and institutional affiliations.

\begin{abstract}
Author details
${ }^{1}$ McFadden BioStress Laboratory, Agronomy, Horticulture, and Plant Science Department, South Dakota State University, Brookings, SD 57006, USA 2JABSOM Bioinformatics Core, Department of Complementary \& Integrative Medicine, University of Hawaii, Honolulu, HI, USA. ${ }^{3}$ South Dakota State University, Brookings, SD 57006, USA. ${ }^{4}$ Department of Biochemistry and Molecular Biology, University of Nevada, Reno, NV, USA
\end{abstract}

Received: 15 February 2018 Accepted: 28 January 2019

\section{Published online: 13 February 2019}

\section{References}

1. OIV. State of vitiviniculture world market. http://www.oiv.int/en/technicalstandards-and-documents/statistical-analysis/state-of-vitiviniculture. 2017. Accessed 31 Jan 2019

2. Pierquet $P$, Stushnoff $C$. Variation and breeding potential of some northern clones of Vitis riparia Michx. Fruit Var J. 1978;32(4):74-84.

3. Carbonneau A. The early selection of grapevine rootstocks for resistance to drought conditions. Am J Enol Viticult. 1985;36:196-8.

4. Fennell A. Freezing tolerance and injury in grapevines. J Crop Improv. 2004; 10:201-35.

5. Galet P. A practical ampelography. New York: Cornell University Press; 1979.

6. Lowe KM, Walker MA. Genetic linkage map of the interspecific grape rootstock cross Ramsey (Vitis champinii)× Riparia Gloire (Vitis riparia). Theor Appl Genet. 2006;112(8):1582-92.

7. Padgett-Johnson M, Williams L, Walker M. Vine water relations, gas exchange, and vegetative growth of seventeen Vitis species grown under irrigated and nonirrigated conditions in California. J Am Soc Hortic Sci. 2003:128:269-76

8. Jones GV, Davis RE. Climate influences on grapevine phenology, grape composition, and wine production and quality for Bordeaux, France. Amer J Enol Vitic. 2000;51:249-61

9. Mozell MR, Thach L. The impact of climate change on the global wine industry: challenges and solutions. Wine Econ Pol. 2014;3:81-9.
10. Tramontini S, Vitali M, Centioni L, Schubert A, Lovisolo C. Rootstock control of scion response to water stress in grapevine. Environ Exp Bot. 2013;93:20-6.

11. Webb LB, Whetton PH, Barlow EWR. Modelled impact of future climate change on the phenology of winegrapes in Australia. Aust J Grape Wine Res. 2007:13:165-75.

12. Gambetta GA, Fei J, Rost TL, Knipfer T, Matthews MA, Shackel KA, Walker MA, McElrone AJ. Water uptake along the length of grapevine fine roots. Developmental anatomy, tissue-specific aquaporin expression, and pathways of water transport. Plant Physiol. 2013;163(3):1254-65.

13. Haider MS, Zhang C, Kurjogi MM, Pervaiz T, Zheng T, Zhang C, Lide C, Shangguan L, Fang J. Insights into grapevine defense response against drought as revealed by biochemical, physiological and RNAseq analysis. Sci Rep. 2017;7(1):13134.

14. Hopper DW, Ghan R, Schlauch KA, Cramer GR. Transcriptomic network analyses of leaf dehydration responses identify highly connected ABA and ethylene signaling hubs in three grapevine species differing in drought tolerance. BMC Plant Biol. 2016;16(1):118.

15. Rattanakon S, Ghan R, Gambetta GA, Deluc LG, Schlauch KA, Cramer GR Abscisic acid transcriptomic signaling varies with grapevine organ. BMC Plant Biol. 2016;16(1):72.

16. Gambetta GA, Manuck CM, Drucker ST, Shaghasi T, Fort K, Matthews MA, Walker MA, McElrone AJ. The relationship between root hydraulics and scion vigour across Vitis rootstocks: what role do root aquaporins play? J Exp Bot. 2012;63(18):6445-55.

17. Rossdeutsch L, Edwards E, Cookson SJ, Barrieu F, Gambetta GA, Delrot S, Ollat N. ABA-mediated responses to water deficit separate grapevine genotypes by their genetic background. BMC Plant Biol. 2016;16(1):91

18. Corso M, Vannozzi A, Maza E, Vitulo N, Meggio F, Pitacco A, Telatin A, D'Angelo M, Feltrin E, Negri AS. Comprehensive transcript profiling of two grapevine rootstock genotypes contrasting in drought susceptibility links the phenylpropanoid pathway to enhanced tolerance. J Exp Bot. 2015;66(19):5739-52.

19. Dodd IC, Stikic R, Davies WJ. Chemical regulation of gas exchange and growth of plants in drying soil in the field. J Exp Bot. 1996;47:1475-90.

20. Chaves MM, Maroco JP, Pereira JS. Understanding plant response to drought - from genes to the whole plant. Func Plant Biol. 2003;30:239-64.

21. Davies WJ, Tardieu F, Trejo CL. How do chemical signals work in plants that grow in drying soil? Plant Physiol. 1994;104(2):309-14.

22. Davies WJ, Kudoyarova G, Hartung W. Long-distance ABA signalling and its relation to other signalling pathways in the detection of soil drying and the mediation of the plant response to drought. J Plant Growth Reg. 2005;24:285-95

23. Liu F, Jensen $C R$, Andersen MN. Hydraulic and chemical signals in the control of leaf expansion and stomatal conductance in soybean exposed to drought stress. Func Plant Biol. 2003;30:65-73.

24. Wilkinson S, Davies WJ. ABA-based chemical signalling: the co-ordination of responses to stress in plants. Plant Cell Environ. 2002;25:195-210.

25. Sobeih WY, Dodd IC, Bacon MA, Grierson D, Davies WJ. Long-distance signals regulating stomatal conductance and leaf growth in tomato (Lycopersicon esculentum) plants subjected to partial rootzone drying. J Exp Bot. 2004;55(407):2353-63.

26. Cramer GR, Ergül A, Grimplet J, Tillett RL, Tattersall EA, Bohlman MC, Vincent D, Sonderegger J, Evans J, Osborne C. Water and salinity stress in grapevines: early and late changes in transcript and metabolite profiles. Func Integr Genom. 2007;7(2):111-34.

27. Cramer GR, SCV S, Hopper DW, Pascovici D, Keighley T, Haynes PA. Proteomic analysis indicates massive changes in metabolism prior to the inhibition of growth and photosynthesis of grapevine (Vitis vinifera L.) in response to water deficit. BMC Plant Biol. 2013;13(1):49.

28. Cramer GR, Urano K, Delrot S, Pezzotti M, Shinozaki K. Effects of abiotic stress on plants: a systems biology perspective. BMC Plant Biol. 2011;11(1): 163.

29. Deluc LG, Quilici DR, Decendit A, Grimplet J, Wheatley MD, Schlauch KA, Mérillon J-M, Cushman JC, Cramer GR. Water deficit alters differentially metabolic pathways affecting important flavor and quality traits in grape berries of cabernet sauvignon and chardonnay. BMC Genomics. 2009:10:212.

30. Chaves MM, Zarrouk O, Francisco R, Costa JM, Santos T, Regalado AP, Rodrigues ML, Lopes CM. Grapevine under deficit irrigation: hints from physiological and molecular data. Annl Bot. 2010;105:661-76. 
31. Escalona JM, Flexas J, Medrano H. Stomatal and non-stomatal limitations of photosynthesis under water stress in field-grown grapevines. Aust J Plant Physiol. 1999;26:421-33.

32. Savoi S, Darren C, Wong J, Arapitsas P, Miculan M, Bucchetti B, Peterlunger E, Fait A, Mattivi F, Castellarin SD. Transcriptome and metabolite profiling reveals that prolonged drought modulates the phenylpropanoid and terpenoid pathway in white grapes (Vitis vinifera L.). BMC Plant Biol. 2016;16:67

33. Griesser M, Weingart G, Schoedl-Hummel K, Neumann N, Becker M, Varmuza K, Liebner F, Schuhmacher R, Forneck A. Severe drought stress is affecting selected primary metabolites, polyphenols, and volatile metabolites in grapevine leaves (Vitis vinifera cv. Pinot noir). Plant Physiol Biochem. 2015;88:17-26.

34. Begg JE, Turner NC. Water potential gradients in field tobacco. Plant Physiol. 1970;46(2):343-6.

35. Mathiason K, He D, Grimplet J, Venkateswari J, Galbraith DW, Or E, Fennell A. Transcript profiling in Vitis riparia during chilling requirement fulfillment reveals coordination of gene expression patterns with optimized bud break. Func Integr Genom. 2009;9:81-96.

36. Pfaffl MW. A new mathematical model for relative quantification in real-time RT-PCR. Nucleic Acids Res. 2001;29(9):e45.

37. Schmieder R, Edwards R. Quality control and preprocessing of metagenomic datasets. Bioinformatics. 2011;27(6):863-4.

38. Subramanian A, Tamayo P, Mootha VK, Mukherjee S, Ebert BL, Gillette MA, Paulovich A, Pomeroy SL, Golub TR, Lander ES, Mesirov JP. Gene set enrichment analysis: a knowledge-based approach for interpreting genomewide expression profiles. PNAS. 2005;102:15545-15550.

39. Subramanian A, Kuehn H, Gould J, Tamayo P, Mesirov JP. GSEA-P: a desktop application for gene set enrichment analysis. Bioinformatics. 2007:23(23):3251-3.

40. Fennell AY, Schlauch KA, Gouthu S, Deluc LG, Khadka V, Sreekantan L, Grimplet J, Cramer GR, Mathiason KL. Short day transcriptomic programming during induction of dormancy in grapevine. Front Plant Sci. 2015;6:834.

41. Grimplet J, Cramer GR, Dickerson JA, Mathiason K, Hemert JV, Fennell AY. VitisNet: "Omics" Integration through Grapevine Molecular Networks. PLOS One. 2009;4(12):e8365.

42. Grimplet J, Hemert JV, Carbonell-Bejerano P, Díaz-Riquelme J, Dickerson J, Fennell A, Pezzotti M, Martínez-Zapater JM. Comparative analysis of grapevine whole-genome gene predictions, functional annotation, categorization and integration of the predicted gene sequences. BMC Res Notes. 2012;5:213.

43. Finkelstein R. Abscisic acid synthesis and response: The Arabidopsis Book; American Society of Plant Biologists. 2013. p. e0166.

44. Schultz HR, Matthews MA. Resistance to water transport in shoots of Vitis vinifera $\mathrm{L}$. relation to growth at low water potential. Plant Physiol. 1988;88:718-24

45. Schultz HR, Matthews MA. Vegetative growth distribution during water deficits in Vitis vinifera L. Aust J Plant Physiol. 1988;15(5):641-56.

46. Dry PR, Loveys BR. Grapevine shoot growth and stomatal conductance are reduced when part of the root system is dried. Vitis. 1999;38(4):151-6.

47. Chabot BF, Hicks DJ. The ecology of leaf life spans. Ann Rev Ecol Syst. 1982;13:229-59.

48. Sapeta H, Lourenço T, Lorenz S, Grumaz C, Kirstahler P, Barros PM, Costa JM, Sohn K, Oliveira MM. Transcriptomics and physiological analyses reveal

co-ordinated alteration of metabolic pathways in Jatropha curcas drought tolerance. J Exp Bot. 2015;67(3):845-60.

49. Tyree MT, Cochard $H$, Cruiziat $P$, Sinclair B, Ameglio T. Drought-induced leaf shedding in walnut: evidence for vulnerability segmentation. Plant Cell Environ. 1993;16(7):879-82.

50. Prinsi B, Negri AS, Failla O, Sciena A, Esperi L. Root proteomic and metabolic analyses reveal specific reesponses to drought stress indifferently tolerant rootstocks. BMC Plant Bio. 2018;18:126.

51. Chaves MM, Pereira JS, Maroco J, Rodriques ML, CPP R, Osório ML, Carvalho I, Faria T, Pinheiro C. How plants cope with water stress in the field. Photosynthesis and growth. Ann Bot. 2002:89:907-16.

52. Rizhsky L, Liang $H$, Mittler R. The combined effect of drought stress and heat shock on gene expression in tobacco. Plant Physiol. 2002;130:1143-51.
53. Alonso R, Berli FJ, Bottini R, Piccoli P. Acclimation mechanisms elicited by sprayed abscisic acid, solar UV-B and water deficit in leaf tissues of field-grown grapevines. Plant Physiol Biochem. 2015;91:56-60.

54. Zong W, Zhong X, You J, Xiong L. Genome-wide profiling of histone H3K4-tri-methylation and gene expression in rice under drought stress. Plant Mol Biol. 2013:81:175-88.

55. Selvaraj MG, Ishizaki T, Valencia M, Ogawa S, Dedicova B, Ogata T, Yoshiwara K, Maruyama K, Kusano M, Saito K. Overexpression of an Arabidopsis thaliana galactinol synthase gene improves drought tolerance in transgenic rice and increased grain yield in the field. Plant Biotech J. 2017;15(11):1465-77.

56. Taji T, Ohsumi C, luchi S, Seki M, Kasuga M, Kobayashi M, Yamaguchi-Shinozaki K, Shinozaki K. Important roles of drought-and cold-inducible genes for galactinol synthase in stress tolerance in Arabidopsis thaliana. Plant J. 2002;29(4):417-26.

57. Medici A, Maryse L, Atanassova R. Profiling of sugar transporter genes in grapevine coping with water deficit. FEBS Lett. 2014;588(21):3989-97.

58. Schmidt A, Hall MN. Signaling to the actin cytoskeleton. Annu Rev Cell Dev Biol. 1998:14:305-38.

59. Chowdhury S, Smith KW, Gustin MC. Osmotic stress and the yeast cytoskeleton: phenotype-specific suppression of an actin mutation. J Cell Biol. 1992;118(3):561-71.

60. Gigon A, Matos A-R, Laffray D, Zuily-Fodil Y, Phan-Thi A-T. Effect of drought stress on lipid metabolism in the leaves of Arabidopsis thaliana (ecotype Columbia). Ann Bot. 2004:94:345-51.

61. Toumi I, Gargouri M, Nouairi I, Moschou PN, Salem-Fnayou AB, Mliki A, Zarrouk M, Ghorbel A. Water stress induced changes in the leaf lipid composition of four grapevine genotypes with different drought tolerance. Biol Plant. 2008;52(1):161-4.

62. Boudière $L$, Michaud $M$, Petroutsos $D$, Rébeillé $F$, Falconet $D$, Bastien $O$, Roy S, Finazzi G, Rolland N, Jouhet J. Glycerolipids in photosynthesis: composition, synthesis and trafficking. Biochim Biophys Acta. 2014;1837(4): 470-80

63. Shaid $\mathrm{S}$, Brandts $\mathrm{CH}$, Serve $\mathrm{H}$, Dikic I. Ubiquitination and selective autophagy. Cell Death Differ. 2013;20(1):21-30.

64. Zhai Y, Guo M, Wang H, Lu J, Liu J, Zhang C, Gong Z, Lu M. Autophagy, a conserved mechanism for protein degradation, responds to heat, and other abiotic stresses in Capsicum annuum L. Front Plant Sci. 2016;7:131.

65. Wang P, Zhao Y, Li Z, Hsu C-C, Liu X, Fu L, Hou Y-J, Du Y, Xie S, Zhang C. Reciprocal regulation of the TOR kinase and $A B A$ receptor balances plant growth and stress response. Mol Cell. 2017;69:100-12.

66. Serra I, Strever A, Myburgh P, Deloire A. Review: the interaction between rootstocks and cultivars (Vitis vinifera L.) to enhance drought tolerance in grapevine. Aust J Grape Wine Res. 2014;20(1):1-14.

67. Blokhina O, Fagerstedt KV. Reactive oxygen species and nitric oxide in plantmitochondria: origin and redundant regulatory systems. Physiol Plant. 2010;138(4):447-62.

68. Heyno E, Mary V, Schopfer P, Krieger-Liszkay A. Oxygen activation at the plasma membrane: relation between superoxide and hydroxyl radical production by isolated membranes. Planta. 2011;234(1):35-45.

69. Sharma $P$, Jha AB, Dubey RS, Pessarakli M. Reactive oxygen species, oxidative damage, and antioxidative defense mechanism in plants under stressful conditions. J Bot. 2012;26:217037.

70. Karuppanapandian T, Moon J-C, Kim C, Manoharan K, Kim W. Reactive oxygen species in plants: their generation, signal transduction, and scavenging mechanisms. Aus J Crop Sci. 2011;5(6):709-25.

71. Salazar-Parra C, Aguirreolea J, Sánchez-Díaz M, Irigoyen JJ, Morales F. Climate change (elevated CO2, elevated temperature and moderate drought) triggers the antioxidant enzymes' response of grapevine $\mathrm{cV}$. Tempranillo, avoiding oxidative damage. Physiol Plant. 2012;144(2):99-110.

72. Marin E, Nussaume L, Quesada A, Gonneau M, Sotta B, Hugueney P, Frey A, Marion-Poll A. Molecular identification of zeaxanthin epoxidase of Nicotiana plumbaginifolia, a gene involved in abscisic acid biosynthesis and corresponding to the ABA locus of Arabidopsis thaliana. EMBO J. 1996;15(10):2331-42.

73. Audran C, Liotenberg S, Gonneau M, North H, Frey A, Tap-Waksman K, Vartanian N, Marion-Poll A. Localisation and expression of zeaxanthin epoxidase mRNA in Arabidopsis in response to drought stress and during seed development. Aust J Plant Physiol. 2001;28:1161-73.

74. Thompson AJ, Jackson AC, Parker RA, Morpeth DR, Burbidge A, Taylor IB. Abscisic acid biosynthesis in tomato: regulation of zeaxanthin epoxidase and 9-cis-epoxycarotenoid dioxygenase mRNAs by light/dark cycles and abscisic acid. Plant Mol Biol. 2000;42:833-45. 
75. Burla B, Pfrunder S, Nagy R, Francisco RM, Lee Y, Martinoia E. Vacuolar transport of abscisic acid glucosyl ester is mediated by ATP-binding cassette and proton-antiport mechanisms in Arabidopsis. Plant Physiol. 2013;163(3):1446-58

76. Lee KH, Piao HL, Kim H-Y, Choi SM, Jiang F, Hartung W, Hwang I, Kwak JM, Lee I-J, Hwang I. Activation of glucosidase via stress-induced polymerization rapidly increases active pools of abscisic acid. Cell. 2006;126(6):1109-20.

77. Jiang F, Hartung W. Long-distance signaling of abscisic acid (ABA): the factors regulating the intensity of the ABA signal. J Exp Bot. 2008;59:37-43.

78. Sauter A, Dietz KJ, Hartung W. A possible stress physiological role of abscisic acid conjugates in root-to-shoot signaling. Plant Cell Environ. 2002;25:223-8.

79. Kuhn JM, Boisson-Dernier A, Dizon MB, Maktabi MH, Schroeder II. The protein phosphatase AtPP2CA negatively regulates abscisic acid signal transduction in Arabidopsis, and effects of abh1 on AtPP2CA mRNA. Plant Physiol. 2006;140:127-39.

80. Saez A, Robert N, Maktabi MH, Schroeder Jl, Serrano R, Rodriguez PL. Enhancement of abscisic acid sensitivity and reduction of water consumption in Arabidopsis by combined inactiviation of the protein phosphatases type 2C, ABI1 and HAB1. Plant Physiol. 2006;141:1389-99.

81. Rubio S, Rodrigues A, Saez A, Dizon MB, Galle A, Kim T-H, Santiago J, Flexas J, Schroeder Jl, Rodriguez PL. Triple loss of function of protein phosphatases type $2 \mathrm{C}$ leads to partial constitutive response to endogenous abscisic acid. Plant Physiol. 2009;150:1345-55.

82. Zandkarimi H, Ebadi A, Salami SA, Alizade H, Baisakh N. Analyzing the expression profile of $A R E B / A B F$ and DREB/CBF genes under drought and salinity stresses in grape (Vitis vinifera L.). PloS One. 2015;10(7):e0134288.

83. Gonzalez-Guzman M, Rodriguez L, Lorenzo-Orts L, Pons C, Sarrion-Perdigones A, Fernandez MA, Peirats-Llobet M, Forment J, Moreno-Alvero M, Cutler SR. Tomato PYR/PYL/RCAR abscisic acid receptors show high expression in root, differential sensitivity to the abscisic acid agonist quinabactin, and the capability to enhance plant drought resistance. J Exp Bot. 2014;65(150):4451-64.

84. Tischer SV, Wunschel C, Papacek M, Kleigrewe K, Hofmann T, Christmann A, Grill E. Combinatorial interaction network of abscisic acid receptors and coreceptors from Arabidopsis thaliana. PNAS. 2017;114(38):10280-5.

85. Wang P, Zhao Y, Li Z, Hsu C-C, Liu X, Fu L, Hou YJ, Xie S, Zhang C, Gao J, Cao M, Huang $X$, Zhu $Y$, Tang $K$, Wang $X$, Tao WA, Xiong $Y$, Zhu JK. Reciprocal regulation of the TOR kinase and ABA receptor balances plant growth and stress response. Mol Cell. 2018;69:100-12.

86. Grundy J, Stoker C, Carré IA. Circadian regulation of abiotic stress tolerance in plants. Front Plant Sci. 2015:6:648.

87. O'Brien JA, Benková E. Cytokinin cross-talking during biotic and abiotic stress responses. Front Plant Sci. 2013;4:451.

88. Tran L-S, Shozaki K, Yamaguchi-Shinozaki K. Role of cytokinin responsive two-component system in ABA and osmotic stress signalings. Plant Signal Behav. 2010;5:148-50.

89. Jeong $Y Y$, Seo PJ. Bidirectional regulation between circadian clock and ABA signaling. Commun Integr Biol. 2017;10:e1296999.

90. Seung D, Risopatron JPM, Jones BJ, Marc J. Circadian clock-dependent gating in ABA signalling networks. Protoplasma. 2012;249(3):445-57.

91. Castells E, Portolés S, Huang W, Mas P. A functional connection between the clock component TOC1 and abscisic acid signaling pathways. Plant Sign Behav. 2010;5(4):409-11.

92. Legnaioli T, Cuevas J, Mas P. TOC1 functions as a molecular switch connecting the circadian clock with plant responses to drought. EMBO J. 2009:28(23):3745-57.

93. Nakamichi N, Takao S, Kudo T, Kiba T, Wang Y, Kinoshita T, Sakakibara H. Improvement of Arabidopsis biomass and cold, drought and salinity stress tolerance by modified circadian clock-associated PSEUDO-RESPONSE REGULATORs. Plant Cell Physiol. 2016:57(5):1085-97.

94. Müller LM, Korff MV, Davis SJ. Connections between circadian clocks and carbon metabolism reveal species-specific effects on growth control. J Exp Bot. 2014;65(11):2915-23.

95. Voß U, Wilson MH, Kenobi K, Gould PD, Robertson FC, Peer WA, Lucas M, Swarup K, Casimiro I, Holman TJ, Wells DM, Peret B, Goh T, Fukaki H, Hodgman TC, Laplaze L, Halliday KJ, Ljung K, Murphy AS, Aj H, AAR W, Bennett MJ. The circadian clock rephases during lateral root organ intiation in Arabidopsis thaliana. Nat Commun. 2015;6:7641.

96. Joshi R, Wani SH, Singh B, Bohra A, Dar ZA, Lone AA, Pareek A, SinglaPareek SL. Transcription factors and plants response to drought stress: current understanding and future directions. Front Plant Sci. 2016;7:1029.
97. Nakashima K, Yamaguchi-Shinozaki K, Shinozaki K. The transcriptional regulatory network in the drought response and its crosstalk in abiotic stress responses including drought, cold, and heat. Front Plant Sci. 2014;5:170.

98. Wang $\mathrm{N}$, Zheng $\mathrm{Y}$, Xin H, Fang L, Li S. Comprehensive analysis of NAC domain transcription factor gene family in Vitis vinifera. Plant Cell Rep. 2013;32(1):61-75.

99. Fang L, Su L, Sun X, Li X, Sun M, Karungo SK, Fang S, Chu J, Li S, Xin H. Expression of Vitis amurensis NAC26 in Arabidopsis enhances drought tolerance by modulating jasmonic acid synthesis. J Exp Bot. 2016;67(9):2829-45.

100. Abe H, Urao T, Ito T, Seki M, Shinozaki K, Yamaguchi-Shinozaki K. Arabidopsis AtMYC2 (bHLH) and AtMYB2 (MYB) function as transcriptional activators in abscisic acid signaling. Plant Cell. 2002;15:63-78.

101. Denekamp M, Smeekens SC. Integration of wounding and osmotic stress signals determines the expression of the AtMYB102 transcription factor gene. Plant Physiol. 2003;132:1415-23.

102. Yang A, Dai X, Zhang W-H. A R2R3-type MYB gene, OsMYB2, is involved in salt, cold, and dehydration tolerance in rice. J Exp Bot. 2012;63(7):2541-56.

103. Pasquali G, Biricolti S, Locatelli F, Baldoni E, Mattana M. Osmyb4 expression improves adaptive responses to drought and cold stress in transgenic apples. Plant Cell Rep. 2008;27:1677-86.
Ready to submit your research? Choose BMC and benefit from:

- fast, convenient online submission

- thorough peer review by experienced researchers in your field

- rapid publication on acceptance

- support for research data, including large and complex data types

- gold Open Access which fosters wider collaboration and increased citations

- maximum visibility for your research: over $100 \mathrm{M}$ website views per year

At $\mathrm{BMC}$, research is always in progress.

Learn more biomedcentral.com/submissions 